Groups Geom. Dyn. 4 (2010), 709-738

DOI $10.4171 / \mathrm{GGD} / 102$
Groups, Geometry, and Dynamics

(C) European Mathematical Society

\title{
Largeness of LERF and 1-relator groups
}

\author{
Jack O. Button
}

\begin{abstract}
We consider largeness of groups given by a presentation of deficiency 1, where the group is respectively free-by-cyclic, LERF or 1-relator. We give the first examples of (finitely generated free)-by- $\mathbb{Z}$ word hyperbolic groups which are large, show that a LERF deficiency 1 group with first Betti number at least two is large or $\mathbb{Z} \times \mathbb{Z}$ and show that 2-generator 1-relator groups where the relator has height 1 obey the dichotomy that either the group is large or all its finite images are metacyclic.
\end{abstract}

Mathematics Subject Classification (2010). 20F05.

Keywords. 1-relator groups, largeness, LERF.

\section{Introduction}

A finitely generated group $G$ is said to be large if it has a finite index subgroup possessing a homomorphism onto a non-abelian free group. This has a range of implications: for instance $G$ is SQ-universal (which means that every countable group is a subgroup of a quotient of $G), G$ has uniformly exponential word growth, $G$ has the largest possible subgroup growth for finitely generated groups (which is of strict type $n^{n}$ ) and $G$ has infinite virtual first Betti number. Here we define the first Betti number $\beta_{1}(G)$ to be the maximum integer $m$ for which there is a surjective homomorphism from $G$ to $\mathbb{Z}^{m}$. The virtual first Betti number of $G$ is the supremum of $\beta_{1}(H)$ where $H$ varies over all the finite index subgroups of $G$. If we restrict ourselves to finitely presented groups and define the deficiency of a finite presentation to be the number of generators minus the number of relators, a well-known result of B. Baumslag and S. J. Pride in [7] is that groups with a presentation of deficiency at least 2 are large.

The results in this paper follow on from [27] where the question considered was which groups with a deficiency 1 presentation are large. Clearly $\mathbb{Z}$ and $\mathbb{Z} \times \mathbb{Z}$ are not, and neither are the soluble Baumslag-Solitar groups BS $(1, n)$, where the BaumslagSolitar group $\mathrm{BS}(m, n)$ has the presentation $\left\langle x, y \mid y x^{m} y^{-1}=x^{n}\right\rangle$. There are other cases, such as BS $(2,3)$, but no more residually finite examples are known (indeed all 
others are "far from being residually finite" in a sense that will be made precise in Section 4).

However in [27] many families of deficiency 1 groups which are all large were found. In particular if $F_{n}$ is the free group of rank $n$ then it was shown that freeby-cyclic groups $F_{n} \rtimes_{\alpha} \mathbb{Z}$ are large for $n \geq 2$ if they contain $\mathbb{Z} \times \mathbb{Z}$, which is equivalent here to not being a word hyperbolic group. The question of whether $F_{n} \rtimes_{\alpha} \mathbb{Z}$ is large in the word hyperbolic case was left open, and up until now not a single large example was known. In Section 2 we show that if $\alpha$ is a reducible automorphism then $F_{n} \rtimes_{\alpha} \mathbb{Z}$ is large provided that free-by-cyclic groups have finite index subgroups with first Betti number at least 2. This was raised by A. Casson in [8] Question 12.16. Although still unknown in the word hyperbolic case, if $\alpha$ is a specific reducible automorphism then we merely require for largeness that two particular free-by-cyclic groups have virtual first Betti number at least 2: these are the one obtained by restricting (a suitable power of) $\alpha$ to the invariant free factor, and the free-by-cyclic group formed by quotienting out the invariant free factor. By taking a specific word hyperbolic group in the literature which is of the form $F_{3} \rtimes_{\alpha} \mathbb{Z}$ and using it to make a reducible automorphism, we obtain Corollary 2.4 which gives the first group of the form $F_{n} \rtimes_{\alpha} \mathbb{Z}$ which is known to be both word hyperbolic and large. It double covers a group with the same properties which has a most succinct presentation: $\left\langle t, a \mid t^{6} a t^{-4} a^{-1} t^{-2} a^{-1}\right\rangle$.

We have mentioned that the property of residual finiteness should increase the chances of a deficiency 1 presentation being large. For instance all groups of the form $F_{n} \rtimes_{\alpha} \mathbb{Z}$ are residually finite. In Section 3 we look at deficiency 1 groups which are LERF (also known as subgroup separable). This is considerably stronger than residual finiteness, so we would expect these groups to be large (with three obvious exceptions). Once again though the problem is finding a finite cover with first Betti number at least 2. We prove in Theorem 3.3 that if $G$ is a LERF group with a presentation of deficiency 1 and has such a finite cover then $G$ is large or the fundamental group of the torus or Klein bottle. A recent result in [47] of D. Kochloukova is used in Theorem 3.1 to show that the only possible exceptions to LERF deficiency 1 groups being large (apart from $\mathbb{Z}$ and these two groups) are word hyperbolic groups of the form $F_{n} \rtimes_{\alpha} \mathbb{Z}$. This at least gives us in Corollary 3.2 that all but these three deficiency 1 LERF groups are SQ-universal, but we are prevented from concluding largeness until Casson's question is settled (at least in the LERF case, but even here this seems open). We also look at the question of a type of Tits alternative for deficiency 1 groups which would say that such a group is either soluble or contains a non-abelian free group. The result in [47] mentioned above very nearly established this, but one case is still to be resolved. We show in Corollary 3.4 that this is true if $G$ has a finite index subgroup with first Betti number at least 2 . However it is not true that all finitely generated subgroups of $G$ will either be virtually soluble or contain a non-abelian free group, and Example 3.5 is such a group which has deficiency exactly 1 .

Another much studied class of groups are those with a 1-relator presentation. The intersection of 1-relator and deficiency 1 groups is the class of 2-generator 1-relator 
presentations. Although groups of this form have strong properties, it is not known which ones are large. Moreover we would like to be able to deduce largeness using only information obtained directly from the presentation rather than needing to know a priori that the group has special properties such as residual finiteness.

In Section 4 we settle this question for a particular class of 2-generator 1-relator presentations. Given any relator in 2 variables we can make a change of basis of $F_{2}$ to $\{a, t\}$ such that the exponent sum of $t$ in the relator is zero. We say that $r$ has height 1 if appearances of $t^{ \pm 1}$ in $r$ are such that $t$ alternates with $t^{-1}$. Although this is a restricted set of relators, it is the case that nearly all of the 2-generator 1-relator groups in the literature which have unusual or nasty properties (we review these in that section) are given by height 1 words.

We establish a major dichotomy of groups $G$ with height 1 presentations in Theorem 4.1 which states that either $G$ is large or all its finite images are metabelian. If $G$ is not a soluble Baumslag-Solitar group but is in the latter case then $G$ contains a non-abelian free group, so is far from being metabelian and hence far from being residually finite. We also apply a famous result of Zelmanov on pro- $p$ groups which allows us in Corollary 4.2 to distinguish between the two cases: $G$ is large if and only if it has a finite index subgroup whose abelianisation requires at least 3 generators, which is a condition that can easily be checked on a computer.

Section 5 is a collection of open questions encountered during the preparation of this work which, although some of these might be well known, we have not found in the standard problem lists. They are all on finitely generated and finitely presented groups and the list begins with the most wide ranging questions and then gradually specialises, ending with unsolved problems that are the most relevant to this paper.

The author would like to thank J. Hillman for bringing his attention to the paper [47].

\section{Reducible free-by-cyclic groups}

A powerful method for proving largeness directly from a given finite presentation is to apply Howie's result which is Theorem A in [44]. This tells us that if there is a homomorphism $\chi$ from a finitely presented group $G$ onto $\mathbb{Z}$ such that the Alexander polynomial $\Delta_{G, \chi}(t) \in \mathbb{Z}\left[t^{ \pm 1}\right]$ relative to $\chi$ is identically zero then $G$ is large (indeed the proof shows that a finite index subgroup containing ker $\chi$ surjects onto a nonabelian free group). However not only do we need the abelianisation $\bar{G}=G / G^{\prime}$ of $G$ to be infinite in order to have a homomorphism onto $\mathbb{Z}$ in the first place, we also have that if $\bar{G}=\mathbb{Z} \times T$ for $T$ finite then $\left|\Delta_{G, \chi}(1)\right|$ divides the order of $T$. Thus we will not be able to use this criterion for largeness unless the first Betti number $\beta_{1}(G)$ is at least two, or we can find a finite index subgroup with this property. (In fact Howie's theorem is also true if the mod $p$ Alexander polynomial $\Delta_{G, \chi}^{p} \in \mathbb{F}\left[t^{ \pm 1}\right]$ for $\mathbb{F}=\mathbb{Z} / p \mathbb{Z}$ is zero and we will use this in later sections, but for now we will stick to the characteristic zero version.) 
Given a finitely presented group $G=\left\langle x_{1}, \ldots, x_{m} \mid r_{1}, \ldots, r_{n}\right\rangle$ which does have a homomorphism $\chi$ onto $\mathbb{Z}$ where $K=\operatorname{ker} \chi$, we can regard $K / K^{\prime}$ as a $\mathbb{Z}\left[t^{ \pm 1}\right]$-module where $t$ acts by conjugation on $K$ using an element of $\chi^{-1}(1)$. Moreover we can obtain a finite presentation for this module $K / K^{\prime}$ by using the Reidemeister-Schreier rewriting process to go from a group presentation for $G$ to a group presentation for $K$, and then abelianising the relations. Although this will result in infinitely many group relations there are only finitely many orbits under the action of $t$. The result is an $(m-1)$ by $n$ presentation matrix $M$ for $K / K^{\prime}$ and we can assume that $n \geq m-1$ by adding zero columns if necessary. We then define the Alexander polynomial $\Delta_{G, \chi} \in \mathbb{Z}\left[t^{ \pm 1}\right]$ to be the highest common factor of the $(m-1)$ by $(m-1)$ minors of $M$; it is the same (up to units in $\mathbb{Z}\left[t^{ \pm 1}\right]$ ) for any finite presentation of $G$.

The fact that a zero Alexander polynomial implies largeness is particularly useful for groups $G$ with a presentation of deficiency 1 because the resulting matrix is square and so we are merely evaluating the determinant to obtain the Alexander polynomial. In particular if we find that one row or column consists entirely of zeros then we immediately conclude largeness. We do have the problem mentioned above that we need $\beta_{1}(G) \geq 2$ for this to happen, however another advantage of deficiency 1 presentations is that for any finite index subgroup $H$ of $G$ (for which we write $H \leq{ }_{f} G$ ) the Reidemeister-Schreier rewriting process results in a deficiency 1 presentation for $H$. As $H$ is large if and only if $G$ is, we can hope that there is a subgroup $H$ with $\beta_{1}(H) \geq 2$.

A large class of deficiency 1 presentations comes from the free-by-cyclic groups: let $F$ be a free group and $\alpha$ an automorphism of $F$. Then we can form the semidirect product (also called the mapping torus) $F \rtimes_{\alpha} \mathbb{Z}$. If $F_{n}$ is the free group of rank $n$ with free basis $x_{1}, \ldots, x_{n}$ then $F_{n} \rtimes_{\alpha} \mathbb{Z}$ has the presentation

$$
\left\langle x_{1}, \ldots, x_{n}, t \mid t x_{1} t^{-1}=\alpha\left(x_{1}\right), \ldots, t x_{n} t^{-1}=\alpha\left(x_{n}\right)\right\rangle .
$$

The following facts are known about free-by-cyclic groups $F \rtimes_{\alpha} \mathbb{Z}$; see [27], Section 5 and references within.

(1) If $F$ is of infinite rank then $F \rtimes_{\alpha} \mathbb{Z}$ may be finitely or infinitely generated. If $F \rtimes_{\alpha} \mathbb{Z}$ is infinitely generated then it need not be large, nor residually finite, but if it is finitely generated then it is residually finite and finitely presented. Moreover it has a presentation with deficiency at least 2, so is large.

(2) $F_{n} \rtimes_{\alpha} \mathbb{Z}$ is residually finite, has deficiency exactly equal to 1 and any finite index subgroup is also of the form $F_{m} \rtimes_{\beta} \mathbb{Z}$, so also has deficiency exactly 1 .

(3) $F_{n} \rtimes_{\alpha} \mathbb{Z}$ is word hyperbolic precisely when it does not contain a subgroup isomorphic to $\mathbb{Z} \times \mathbb{Z}$. If it does and $n \geq 2$ then it is large. However it is not known whether word hyperbolic groups of the form $F_{n} \rtimes_{\alpha} \mathbb{Z}$ are large. A problem in [8] due to Casson is whether a group $G=F_{n} \rtimes_{\alpha} \mathbb{Z}$ with $n \geq 2$ always has $H \leq_{f} G$ with $\beta_{1}(H) \geq 2$. Whilst this is true if $G$ contains $\mathbb{Z} \times \mathbb{Z}$, it is unknown in general if $G$ is word hyperbolic and so we will need to take this as an assumption. 
An automorphism $\alpha$ of the free group $F_{n}$ for $n \geq 2$ is said to be reducible if there exist proper non-trivial free factors $R_{1}, \ldots, R_{k}$ of $F_{n}$ such that the conjugacy classes of $R_{1}, \ldots, R_{k}$ are permuted transitively by $\alpha$ (see [11]).

Theorem 2.1. Assume that any group of the form $F_{n} \rtimes_{\alpha} \mathbb{Z}$ for $n \geq 2$ has a finite index subgroup with first Betti number at least 2. Then if $G=F_{n} \rtimes_{\alpha} \mathbb{Z}$ for $\alpha$ a reducible automorphism, we have that $G$ is large.

Proof. Our group $G$ will have a presentation as in (1) and so for each positive integer $k$ there exists the cyclic cover of $G$ which is the index $k$ subgroup $F_{n} \rtimes_{\alpha^{k}} \mathbb{Z}$ generated by $t^{k}$ and $F_{n}$. If $\alpha$ is reducible then on replacing $\alpha^{k}$ by $\alpha$ we can assume $F_{n}=A * B$ with $A$ and $B$ proper free factors and $\alpha(A)$ is sent to a conjugate of $A$. However on now composing $\alpha$ with an appropriate inner automorphism (which does not change the free-by-cyclic group) we can assume that $\alpha(A)=A$. From now on this will be our $G$.

We define the free-by-cyclic group $G_{r}$ by restricting $\alpha$ to $A$. Our assumption means that we can take a finite index subgroup of $G_{r}$ with first Betti number at least 2 (and note that if $A$ has rank 1 then we can do this as well). But every finite index subgroup of $F_{n} \rtimes_{\alpha} \mathbb{Z}$ contains one of the form $F_{m} \rtimes_{\alpha^{k}} \mathbb{Z}$ where $F_{m} \leq_{f} F_{n}$ and $\alpha^{k}\left(F_{m}\right)=F_{m}$. Moreover the first Betti number does not decrease in finite covers. Doing this for $G_{r}$, we have $A_{0} \leq_{f} A$ and a power of $\alpha$ fixing $A_{0}$ (which we again replace by $\alpha$ ) to get a finite index subgroup $L=\left\langle t, A_{0}\right\rangle$ of $G_{r}$ with $\beta_{1}(L) \geq 2$. However a result of Marshall Hall, Jr., states that as $A_{0}$ is a finitely generated subgroup of $F_{n}=A * B$, there is a finite index subgroup $E$ of $F_{n}$ with $A_{0}$ a free factor, so we have $E=A_{0} * S$ for some $S$ and, as $A_{0}$ does not have finite index in $F_{n}, S$ is non-trivial. Again taking a power of $t$, we can assume that $\alpha^{j}(E)=E$ as there are only finitely many subgroups in $F_{n}$ of each finite index.

We are now ready to look at a finite presentation for the finite index subgroup $J=\left\langle t^{j}, E\right\rangle$ of $G$, which we obtain by first examining a suitable finite presentation for $L=\left\langle t, A_{0}\right\rangle$. Let us take a free basis $a_{1}, \ldots, a_{l}$ for $A_{0}$, where $l$ is the rank of $A_{0}$, and a similar one for $S$. Let $\bar{\alpha}$ be the automorphism of $\mathbb{Z}^{l}$ induced by $\alpha$ when $A_{0}$ is abelianised. Because $\beta_{1}(L) \geq 2$ we must have a non-zero $u \in \mathbb{Z}^{l}$ such that $\bar{\alpha}(u)=u$, and so we can extend $u$ (or $u / n$ for some positive integer $n$ ) to a basis for $\mathbb{Z}^{l}$. Now we can find an automorphism of $A_{0} \cong F_{l}$ sending the basis $a_{1}, \ldots, a_{l}$ to $x_{1}, \ldots, x_{l}$ where $x_{1}$ goes to $u$ under abelianising. Thus $\alpha\left(x_{1}\right)=x_{1} c\left(x_{1}, \ldots, x_{l}\right)$ where $c$ is a word in the commutator subgroup $F_{l}^{\prime}$ of $F_{l}$, and the same is true (with a different $c$ ) for any power of $\alpha$. Therefore on setting $\tau=t^{j}$ we obtain a deficiency 1 presentation for $J$ of the form in (1) where the first $l$ relations are of the form $\tau x_{i} \tau^{-1}=w_{i}\left(x_{1}, \ldots, x_{l}\right)$ where $w_{i}$ are reduced words in $F_{l}$.

Now suppose there exists a surjective homomorphism $\chi$ from $J$ to $\mathbb{Z}$ with the property that $\tau$ and all of $A_{0}$ are in ker $\chi$. When we form the square matrix $M$ with entries in $\mathbb{Z}\left[x^{ \pm 1}\right]$ in order to calculate the polynomial $\Delta_{J, \chi}(x)$, all the letters appearing in the first relation are in the kernel of $\chi$. Therefore we have that the 
first column of $M$ consists in turn of the exponent sum of $\tau, x_{1}, \ldots, x_{l}$ and then zeros in the other places because the latter rows correspond to generators in this presentation for $J$ which do not even appear in the first relation. However the exponent sums of the generators $\tau, x_{1}, \ldots, x_{l}$ that do appear are all zero so we have a zero column in a square matrix, meaning that $\Delta_{J, \chi}$ is the zero polynomial so $J$, and $G$, are large.

In order to find such a homomorphism, we must again use our assumption on finite covers with first Betti number at least 2 . From $G$ we obtained the "reduced" free-by-cyclic group $G_{r}$ by restricting $\alpha$ to $A$. Although the definition of a reducible automorphism means that we cannot assume $\alpha(B)=B$ even if $\alpha(A)=A$, we can form the "quotient" free-by-cyclic group $G_{q}$ by taking the "quotient" automorphism $\tilde{\alpha}$ of $B$. This is formed by letting $\pi: A * B \rightarrow B$ be the homomorphism with kernel the normal closure of $A$ and then we define $\tilde{\alpha}(b)=\pi \alpha(b)$ for $b \in B$. Note that $\tilde{\alpha}$ is surjective because $\alpha$ and $\pi$ are, and the Hopfian property of finitely generated free groups means that $\tilde{\alpha}$ is an automorphism. Also there is a natural homomorphism $\theta$ from $G$ to $G_{q}=\langle s, B\rangle$ given by sending $t$ to $s$ and "ignoring" $A$; this is well defined because $\alpha(A)=A$.

Now we apply our assumption to obtain a finite index subgroup $H$ of $G_{q}$ with $\beta_{1}(H) \geq 2$ and without loss of generality we can assume $H$ is of the form $\left\langle s^{i}, C\right\rangle$ for $C \leq_{f} B$ and some $i \in \mathbb{N}$. In fact as cyclic covers of $H$ will also have first Betti number at least 2, we can replace $i$ with $j$ by taking multiples so that they are both equal to $i j$. As $\beta_{1}(H) \geq 2$, we must have a homomorphism $\tilde{\chi}$ from $H$ onto $\mathbb{Z}$ with $\tilde{\chi}\left(s^{j}\right)=0$. We then consider the subgroup of $G$ which is $\theta^{-1}(H) \cap J$. As each of these subgroups has finite index in $G$, so does their intersection. We can take our finite presentation above for $J$ and use this to rewrite for the finite index subgroup $\theta^{-1}(H) \cap J$. Note that $t^{j}$ is in $\theta^{-1}(H)$ and this presentation will also have deficiency 1 . Also the generators that we used for $A_{0}$ are in $\operatorname{ker} \theta$ so are in $\theta^{-1}(H)$ too. Hence in going from our old presentation to our new one, our valuable relation survives intact. But the homomorphism $\tilde{\chi} \theta: \theta^{-1}(H) \rightarrow \mathbb{Z}$ can be restricted to $\theta^{-1}(H) \cap J$ which has finite index in $\theta^{-1}(H)$. Without loss of generality this restriction is surjective and moreover all the generators in our special relation end up being sent to the identity by $\tilde{\chi} \theta$, so we are done.

Corollary 2.2. Assume that any group $F_{n} \rtimes_{\alpha} \mathbb{Z}$ for $n \geq 2$ has a finite index subgroup with first Betti number at least two. Suppose that the group $G$ can be written in the form $F_{n} \rtimes_{\alpha} \mathbb{Z}$ and there exists a finitely generated subgroup $A$ of $F_{n}$ which is nontrivial and of infinite index in $F_{n}$, whose conjugacy class has a finite orbit under $\alpha$. Then $G$ is large.

Proof. By taking cyclic covers and an inner automorphism, we can assume that $\alpha(A)=A$. Although $A$ may not be a free factor of $F_{n}$, we can again use Marshall Hall's result to find $S$ (with $A$ and $S$ non-trivial) such that $A * S=F_{m}$ and $F_{m}$ has finite index in $F_{n}$. We now take the appropriate power $\alpha^{i}$ of $\alpha$ that fixes $F_{m}$. Then 
the subgroup $H=F_{m} \rtimes_{\alpha^{i}} \mathbb{Z}$ has finite index in $G$, with $\alpha^{i}$ reducible when restricted to $F_{m}$, so $H$ is large by Theorem 2.1.

However we have not seen a single example of a word hyperbolic group of the form $F_{n} \rtimes_{\alpha} \mathbb{Z}$ which is large. Although it seems as if we need to wait for Casson's question on finite index subgroups with first Betti number at least two to be settled positively, we can manage without this in specific cases. If we have an automorphism $\alpha$ of $F_{n}=A * B$ with $\alpha(A)=A$, where $A$ and $B$ are proper free factors, and we form the free-by-cyclic group $G$ then the proof of Theorem 2.1 was set out so that it is enough to find finite index subgroups with first Betti number 2 of both the restriction $G_{r}=A \rtimes_{\alpha} \mathbb{Z}$ and the quotient $G_{q}=B \rtimes_{\tilde{\alpha}} \mathbb{Z}$. If we have a presentation for $G$ as in (1) and our free basis for $F_{n}$ is obtained by putting together ones for $A$ and $B$ then we instantly get finite presentations for $G_{r}$ and $G_{q}$ which we can then feed into a computer and ask it to enumerate finite index subgroups and their abelianisations.

This allows us to check largeness of free-by-cyclic groups $G$ formed by reducible automorphisms $\alpha$. If we require a word hyperbolic example then it is necessary that $G$ contains no $\mathbb{Z} \times \mathbb{Z}$ subgroup, or equivalently $\alpha$ has no periodic conjugacy classes. Moreover this is sufficient for a group of the form $F_{n} \rtimes \mathbb{Z}$ to be word hyperbolic by [9], [10] and [19]. Thus it is straightforward to create reducible word hyperbolic examples by a "doubling" process.

Lemma 2.3. If $A \rtimes_{\alpha} \mathbb{Z}$ is word hyperbolic where $A$ is isomorphic to $F_{n}$ and $\alpha$ is an automorphism of $A$ then $(A * B) \rtimes_{\alpha} \mathbb{Z}$ is also word hyperbolic, where $B$ is a copy of $A$ and the action of $\alpha$ on $B$ is the same as on $A$.

Proof. Suppose we have $w \in A * B$ with $\alpha^{k}(w)$ equal to a conjugate of $w$ for $k \geq 1$. Then $w$ is not in $A$ or $B$, so we can ensure (by conjugation in $A * B$ if necessary) that $w=a_{1} b_{1} \ldots a_{n} b_{n}$ where $a_{i} \in A-\{e\}$ and $b_{i} \in B-\{e\}$. Now on setting $a_{i}^{\prime}=\alpha^{k}\left(a_{i}\right)$ which is in $A$ and $b_{i}^{\prime}=\alpha^{k}\left(b_{i}\right)$ in $B$, we have that $w$ and $a_{1}^{\prime} b_{1}^{\prime} \ldots a_{n}^{\prime} b_{n}^{\prime}$ are conjugate, with both words cyclically reduced in a free group, meaning that there must be $j$ so that (taking subscripts modulo $n$ ) $a_{i}^{\prime}=a_{i+j}$ and $b_{i}^{\prime}=b_{i+j}$. But then $\alpha^{k n}\left(a_{i}\right)=\left(a_{i}\right)$, giving a $\mathbb{Z} \times \mathbb{Z}$ subgroup in $A \rtimes_{\alpha} \mathbb{Z}$.

Note that in this case if $G=(A * B) \rtimes_{\alpha} \mathbb{Z}$ then both $G_{r}$ and $G_{q}$ are isomorphic to the original group $A \rtimes_{\alpha} \mathbb{Z}$.

We now need to find explicit examples of word hyperbolic groups of the form $F_{n} \rtimes_{\alpha} \mathbb{Z}$. To achieve this we use results in [39] and [66]. An automorphism $\alpha$ of $F_{n}$ gives rise to an automorphism of the abelianisation $\mathbb{Z}^{n}$ of $F_{n}$ which is well defined for outer automorphisms, as is the definition of reducibility of $\alpha$. In the former paper Corollary 2.6 states that if we have an outer automorphism $O$ of $F_{n}$ such that the characteristic polynomial of the induced automorphism of $\mathbb{Z}^{n}$ is a PVpolynomial (which means that the polynomial is monic and has exactly one root with modulus greater than one (counted with multiplicity) and no roots on the unit circle) then $O^{k}$ is irreducible for $k \geq 1$. They then obtain as Corollary 2.8 that if $\alpha$ is an 
automorphism of $F_{n}$ for $n \geq 3$ where the corresponding characteristic polynomial is a PV-polynomial then $\alpha(w)=w$ implies that $w=e$. Moreover $\alpha$ can have no periodic conjugacy classes because $\alpha^{k}$ will also have the same property, and if $\alpha$ (or $\alpha^{k}$ ) sends $w$ to a conjugate then we multiply $\alpha$ (or $\alpha^{k}$ ) by an inner automorphism which does not change the characteristic polynomial.

The latter corollary is proved by applying [11], Theorem 4.1, which states that if $O^{k}$ is irreducible for $k \geq 1$ and $O$ fixes a conjugacy class then $O$ is geometrically realised by a pseudo-Anosov homeomorphism of a compact surface with one boundary component. But [66] shows that automorphisms of $F_{n}$ with a characteristic polynomial that is a PV-polynomial are not geometrically realisable for $n \geq 3$ by considering the eigenvalues (note that $F_{2} \rtimes_{\alpha} \mathbb{Z}$ is never word hyperbolic because the commutator of the generators gives rise to a conjugacy class that is fixed or of period 2). An example is given: $G_{0}=F_{3} \rtimes_{\alpha} \mathbb{Z}$ where $\alpha(x)=y, \alpha(y)=z$, $\alpha(z)=x y$.

Corollary 2.4. The $F_{6}$-by- $\mathbb{Z}$ group

$$
\begin{aligned}
G=\langle t, a, b, c, x, y, z| t a t^{-1} & =b, t b t^{-1}=c, t c t^{-1}=a b, \\
t x t^{-1} & \left.=y, t y t^{-1}=z, t z t^{-1}=x y\right\rangle
\end{aligned}
$$

is word hyperbolic and large.

Proof. That $G$ is word hyperbolic follows from the facts quoted above: namely $G_{0}=F_{3} \rtimes_{\alpha} \mathbb{Z}$ has no periodic conjugacy classes, so Lemma 2.3 shows that $G$ also has no periodic conjugacy classes, thus is word hyperbolic. That $G$ is large follows from applying the proof of Theorem 2.1 to $G$ with $G_{r}=G_{q}=G_{0}$, and from the output of a computer. Inputting the presentation of $G_{0}$ into MAGMA and asking for the abelianisation of its low index subgroups, we find (after a bit of a wait, although it is more quickly checked) that $G_{0}$ has an index 14 subgroup (with generators $x, y$, $z^{2}, z x z^{-1}, z y z^{-1}, z t^{-7}$ ) with abelianisation $C_{2} \times C_{4} \times \mathbb{Z} \times \mathbb{Z}$.

We also note that $G$ is the double cover of the automorphism which sends in turn $a$ to $x$ to $b$ to $y$ to $c$ to $z$ to $a b$. Here all other generators except $t$ and $a$ can be eliminated to get the 2-generator 1-relator $F_{6}$-by- $\mathbb{Z}$ word hyperbolic large group $\left\langle t, a \mid t^{6} a t^{-4} a^{-1} t^{-2} a^{-1}\right\rangle$.

\section{LERF groups of deficiency 1}

Not all groups with a deficiency 1 presentation can be large, as evidenced by the Baumslag-Solitar groups $\mathrm{BS}(m, n)=\left\langle a, t \mid t a^{m} t^{-1}=a^{n}\right\rangle$ for $m, n$ non-zero integers, where we can take without loss of generality $m>0$ and $|n| \geq m$. We have that $\mathrm{BS}(m, n)$ is large if and only if $m$ and $n$ are not coprime. For $m=1$ we have a soluble group (and it is known that a virtually soluble group of deficiency 1 
must be isomorphic to $\mathrm{BS}(1, n)$ or to $\mathbb{Z})$, but otherwise $\mathrm{BS}(m, n)$ contains a nonabelian subgroup, thus it is not true that Baumslag-Solitar groups are either virtually soluble or large. However most of these groups are not residually finite (for this we require $m=1$ or $m=|n|$ ) and so if we stick to residually finite Baumslag-Solitar groups, we do have this dichotomy. Thus we could consider residually finite groups of deficiency 1: in fact we know of no example of such a group which is not virtually soluble but not large.

In order to make progress we impose an even tighter condition on our deficiency 1 group $G$, which is that it is LERF (locally extended residually finite, also known as subgroup separable). This means that every finitely generated subgroup is an intersection of finite index subgroups. The big advantage of this is a result of Lubotzky in [51] that if a LERF group $G$ can be written as an HNN extension $H *_{\phi}$ which is non-ascending, that is both the domain $A$ of $\phi$ and the image $B$ are strictly contained in $H$, then $G$ is large if $A$ is finitely generated. This is because $G$ surjects to an HNN extension of a finite group which is virtually free, and the LERF property applied to $A$ means that this HNN extension will also be non-ascending, thus it is virtually free but not virtually cyclic.

Theorem 3.1. If $G$ is LERF and of deficiency 1 then either $G$ is large or $G$ is of the form $F_{n} \rtimes \mathbb{Z}$.

Proof. As there must be a surjective homomorphism $\chi$ from $G$ to $\mathbb{Z}$, we have by [15] that the finitely presented group $G$ is an HNN extension of a finitely generated group $H$ with finitely generated associated subgroups $A$ and $B$. We say that an HNN extension is ascending if at least one of $A$ or $B$ is equal to the base $H$, and it is strictly ascending if exactly one is. We then have that if this HNN extension is not ascending then $G$ is large by the above. But if say $H=A$ then $G=\langle t, H\rangle$ where $t$ is the stable letter and we have $t H t^{-1} \subseteq H$. By a result of Blass and P. M. Neumann, a LERF group cannot have a finitely generated subgroup conjugate to a proper subgroup of itself, so $t H t^{-1}=H$ and we conclude that $H \unlhd G$ with $G=H \rtimes \mathbb{Z}$. Now we can use a recent result of Kochloukova. We have by [42] Theorem 6 that if $G$ has deficiency 1 and is an ascending HNN extension of a finitely generated group then $G$ has geometric (hence cohomological) dimension at most 2. But Theorem 3 in [47] is as follows: Let $G$ be a non-trivial group with a finite $\mathrm{K}(G, 1) \mathrm{CW}$-complex of dimension $n$ with Euler characteristic 0. Suppose that $N$ is a normal subgroup of $G$ containing $G^{\prime}$ which is of homological type $\mathrm{FP}_{n-1}$ and $G / N$ is cyclic-by-finite. Then $N$ is of type $\mathrm{FP}_{n}$. Consequently we conclude by putting $H$ equal to $N$ that $H$ is of type $\mathrm{FP}_{2}$, but a result of Bieri which is Corollary 8.6 in [12] gives us that the cohomological dimension of $H$ is 1 and thus $H$ is free.

It should be noted that groups of the form $F_{n} \rtimes \mathbb{Z}$ are not necessarily LERF. The first example is $\left\langle t, a, b \mid t a t^{-1}=a b, t b t^{-1}=b\right\rangle$ due to Burns, Karrass and Solitar in [24] and is also famous for being the first 3-manifold group known not to be LERF. Also Corollary 3 of [55] gives further examples. 
Consequently if we had that $F_{n} \rtimes \mathbb{Z}$ was large for $n \geq 2$ we would have the strongest possible result: either a deficiency 1 LERF group is large or it is $\mathbb{Z}, \mathbb{Z} \times \mathbb{Z}$ or the Klein bottle group BS $(1,-1)$. However we can move in two directions from this result. The first is to relax the property of largeness to that of $G$ being SQuniversal (every countable group is a subgroup of a quotient of $G$ ) which in turn is stronger than containing the free group $F_{2}$. As groups of the form $F_{n} \rtimes \mathbb{Z}$ for $n \geq 2$ are either large by [27] or hyperbolic, hence SQ-universal by [62], we immediately get:

Corollary 3.2. A LERF group of deficiency 1 is either SQ-universal or is one of $\mathbb{Z}$, $\mathbb{Z} \times \mathbb{Z}, \mathrm{BS}(1,-1)$.

A conjecture of P. M. Neumann from 1973 is that there is a dichotomy for any 1-relator group: either it is SQ-universal or it is a soluble Baumslag-Solitar group or $\mathbb{Z}$. Of course if there are at least 3 generators then later it was shown that we have largeness, but the conjecture remains open in the 2-generator 1-relator case. Corollary 7.5 in [27] showed this to be true if the group is LERF, so in light of Corollary 3.2 we wonder if P. M. Neumann's conjecture extends to all deficiency 1 groups, or (more cautiously) to all residually finite deficiency 1 groups.

The other improvement that we can make to Theorem 3.1 is to give further conditions guaranteeing largeness. It might not be a surprise in light of Section 2 that once again it comes down to requiring first Betti number at least two.

Theorem 3.3. If $G$ is LERF, of deficiency 1 and has a finite index subgroup with first Betti number at least 2 then $G$ is large or $\mathbb{Z} \times \mathbb{Z}$ or the Klein bottle group.

Proof. We assume that $\beta_{1}(G)=b \geq 2$ and is LERF (which is a property preserved by all subgroups). We consider the BNS (Bieri-Neumann-Strebel) invariant $\Sigma$ of $G$ which is an open subset of $S^{b-1}$ and which gives information on the finite generation of kernels of non-trivial homomorphisms from $G$ to $\mathbb{Z}$. The definition of $\Sigma$ is given in the introduction of [14], but we will only need to look at the rationally defined points of $S^{b-1}$. We can regard these rationally defined points as equivalence classes of (nontrivial) homomorphisms $\chi, \chi^{\prime}$ from $G$ to $\mathbb{Z}$ according to the relation $\chi=q \chi^{\prime}$ for $q \in \mathbb{Q}$ and $q>0$. Then by [14], Proposition 4.3, we have that $[\chi]$ is in $\Sigma$ if and only if $G$ can be expressed as an ascending HNN extension $\langle t, H\rangle$, as defined in Theorem 3.1, with associated homomorphism $\chi$ (that is $\chi(t)=1$ and $\chi(H)=0$ ) and with $H$ finitely generated. Moreover ker $\chi$ is finitely generated if and only if $[\chi]$ and $[-\chi]$ are both in $\Sigma$. Now for a LERF group $G$, if there exists $\chi$ with neither of $[ \pm \chi]$ in $\Sigma$ then $G$ is large, as in Theorem 3.1. Otherwise one of $[ \pm \chi]$ is in $\Sigma$ for every homomorphism $\chi$ from $G$ onto $\mathbb{Z}$. But as the LERF condition means that we cannot have $G$ equal to a strictly ascending $H N N$ extension $\langle t, H\rangle$ where $H$ is finitely generated, we must have both $[ \pm \chi] \in \Sigma$ for all possible $\chi$.

Now we use a result of Dunfield in [30]. One can regard the (multivariable) Alexander polynomial $\Delta_{G}\left(t_{1}, \ldots, t_{b}\right)$ as a finite set of lattice points in $\mathbb{Z}^{b}$ labelled 
with a non-zero integer by taking the monomials in $\Delta_{G}$ with non-zero coefficient. We can then form the Newton polytope $N\left(\Delta_{G}\right) \subseteq \mathbb{R}^{b}$ which is the convex hull of these points. Then [30] Theorem 5.1 states that if $D\left(\Delta_{G}\right)$ is the dual of $N\left(\Delta_{G}\right)$ in $\mathbb{R}^{b}$ (so that faces of dimension $i$ become faces of dimension $b-i-1$ ) and $F_{1}, \ldots, F_{k}$ are the $(b-1)$-dimensional faces of $D\left(\Delta_{G}\right)$ whose corresponding vertices of $N\left(\Delta_{G}\right)$ have coefficient \pm 1 then $\Sigma$ is contained in the projection of the interiors of the $F_{i}$ to $S^{b-1}$.

So far we have not used the fact that $b \geq 2$. Now when $b=1$ we get $S^{0}=\{ \pm 1\}$ and the result above is saying that if $\chi$ is the unique surjective homomorphism (up to sign) from $G$ to $\mathbb{Z}$ then ker $\chi$ being finitely generated implies that the highest and lowest terms of $\Delta_{G}(t)$ are monic. However if $b \geq 2$ then the fact that $[\chi] \in \Sigma$ for all homomorphisms $\chi$ implies that $\Delta_{G}=1$. This is because if there are $n \geq 2$ vertices of $N\left(\Delta_{G}\right)$ then we have $(b-1)$-dimensional faces $F_{1}, \ldots, F_{n}$ of $D\left(\Delta_{G}\right)$, and so when we project the interiors of those faces which are obtained from the \pm 1 coefficients, we do not cover all of $S^{b-1}$ because we miss the lower dimensional faces where pairs of elements of $F_{1}, \ldots, F_{n}$ meet. Moreover there will be rationally defined points which are not covered and therefore homomorphisms $\chi$ with $[\chi]$ not in $\Sigma$.

Hence if $G$ is LERF and has $\beta_{1}(G) \geq 2$ then the only way that $G$ fails to be large is if $\Delta_{G}=1$, with ker $\chi$ being finitely generated for all homomorphisms $\chi$ onto $\mathbb{Z}$. However by [25], Theorem 3.1, we have for deficiency 1 groups that

$$
\Delta_{G, \chi}(t)=(t-1) \Delta_{G}\left(t_{1}^{n_{1}}, \ldots, t_{b}^{n_{b}}\right)
$$

where $n_{i}$ is the image under $\chi$ of any element in $G$ which projects to $t_{i}$ under the natural homomorphism from $G$ to its free abelianisation $\mathbb{Z}^{b}=\left\langle t_{1}, \ldots, t_{b}\right\rangle$. Consequently if $\Delta_{G}=1$ then for any $\chi$ we have $\Delta_{G, \chi}$ equal to $t-1$. But the degree of $\Delta_{G, \chi}$ is the dimension of the $\mathbb{Q}$-vector space $H_{1}(\operatorname{ker} \chi ; \mathbb{Q})=H_{1}(\operatorname{ker} \chi ; \mathbb{Z}) \otimes_{\mathbb{Z}} \mathbb{Q}$, for which we write $\beta_{1}(\operatorname{ker} \chi ; \mathbb{Q})$, and from [47] Theorem 3 we have that ker $\chi$ is free, so it must be free of rank 1 and therefore $G=\mathbb{Z} \times \mathbb{Z}$.

Finally if $G$ has deficiency 1 and has a finite index subgroup $H=\mathbb{Z} \times \mathbb{Z}$ then $G$ is either $\mathbb{Z} \times \mathbb{Z}$ or the Klein bottle group.

We will see in Section 4 that this theorem is not true if the LERF condition is removed. If it is weakened to residually finite then this is unknown.

We can also finish off the proof without needing [47], Theorem 3, by considering the Alexander polynomial $\Delta_{G, \chi}^{p}$ over $\mathbb{Z} / p \mathbb{Z}$. Although it is not true for a general finite presentation, for the deficiency 1 case this is just the Alexander polynomial $\Delta_{G, \chi}$ over $\mathbb{Z}$ reduced modulo $p$. Consequently we must have $\beta_{1}(\operatorname{ker} \chi ; \mathbb{Z} / p \mathbb{Z})=1$ and so $\beta_{1}(G ; \mathbb{Z} / p \mathbb{Z}) \leq 2$. But if this is true for all finite index subgroups $H$ of $G$ then $H / H^{\prime}=\mathbb{Z} \times \mathbb{Z}$ which cannot happen if $G$ is residually finite by [27], Proposition 3.4, unless $G=\mathbb{Z} \times \mathbb{Z}$.

As for attempting to apply the above techniques to deficiency 1 groups which are not LERF, we can squeeze out results on when a deficiency 1 group contains a non-abelian free group (or equivalently contains $F_{2}$ ). In [67] J. S. Wilson conjectures 
that if a finitely presented group $G$ is such that $\operatorname{def}(G)+d^{2} / 4-d>0$, where $d$ is the minimum number of generators for $G / G^{\prime}$ (and assumed to be at least 2), then $G$ contains a non-abelian free group. (Ershov has recently proved in [35] that such a group is non-amenable.)

For $\operatorname{def}(G)=1$ this was established in [44], as Corollary 2.4 in that paper states that if $G$ is of deficiency 1 and $N \unlhd G$ is such that $G / N$ is non-trivial and free abelian with $N / N^{\prime} \otimes_{\mathbb{Z}} \mathbb{F}$ having dimension at least 2 for some field $\mathbb{F}$, then $G$ contains $F_{2}$. Therefore on putting $N=\operatorname{ker} \chi$ for any $\chi$, we have that $\beta_{1}(G ; \mathbb{Z} / p \mathbb{Z})$ is at least 3 for some prime $p$, which implies that $\beta_{1}(N ; \mathbb{Z} / p \mathbb{Z}) \geq 2$.

We also have

Corollary 3.4. If $G$ has deficiency 1 and virtual first Betti number at least 2 then $G$ contains $F_{2}$ or is $\mathbb{Z} \times \mathbb{Z}$ or the Klein bottle group.

Proof. By [14] Theorem D, if a finitely presented group $G$ does not contain $F_{2}$ but $\beta_{1}(G) \geq 2$ then there exists $K=\operatorname{ker} \chi$ which is finitely generated and such that $G / K \cong \mathbb{Z}$. Consequently by [47], Theorem 3, mentioned above, we have that $K$ is of type $\mathrm{FP}_{2}$ and hence free as before. Thus we are done unless $K$ is free of rank 0 (but then $G=\mathbb{Z}$ so its virtual first Betti number is 1 ) or free of rank 1, giving the two exceptions.

In fact it is widely believed that if $G$ has deficiency 1 then it contains $F_{2}$ unless $G=\mathrm{BS}(1, n)$. It is clear that the only case left is when $\beta_{1}(G)=1$ with $1 \leq$ $d\left(G / G^{\prime}\right) \leq 2$, where $d$ is the minimum number of generators of a finitely generated group, and $G$ is a strictly ascending $\mathrm{HNN}$ extension, so that $S^{0}$ contains two points with $\Sigma$ one of them. The conjecture at the end of Section 2 in [13] is that in this case $G$ is a strictly ascending HNN extension with base a finitely generated free group. The BNS invariant $\Sigma$ of a finitely generated group $G$ was later incorporated as one of a family of $n$ dimensional invariants $\Sigma^{n}(G ; \mathbb{Z})$ with $\Sigma=\Sigma^{1}(G ; \mathbb{Z})$. However it is shown in this paper that $\Sigma=\Sigma^{2}(G ; \mathbb{Z})$ if $G$ is a finitely presented group. Therefore we would be done if we knew that having a rationally defined $\chi$ with $[\chi] \in \Sigma^{2}(G ; \mathbb{Z})$ implies that $G$ is an ascending $\mathrm{HNN}$ extension over a base group of type $\mathrm{FP}_{2}$ with associated homomorphism $\chi$. This would be the equivalent for $n=2$ of the BieriNeumann-Strebel result that $[\chi] \in \Sigma=\Sigma^{1}(G ; \mathbb{Z})$ implies that the base is of type $\mathrm{FP}_{1}$, i.e. finitely generated. In the special case of 2-generator 1-relator groups the conjecture above is known: indeed in [53], Chapter II, Section 5, we have a "Tits alternative" which says that a subgroup of a 1-relator group either contains $F_{2}$ or is soluble (whereupon it is locally cyclic, $\mathrm{BS}(1, n)$ or infinite dihedral). However we will not get such an alternative for arbitrary subgroups of deficiency 1 groups, or even arbitrary finitely generated subgroups:

Example 3.5. Thompson's group $T$ has a finite presentation of two generators and two relators but has the unusual property that it does not contain $F_{2}$ nor is it virtually 
soluble. The group $G=T * \mathbb{Z}$ has deficiency at least one, and in fact it is exactly 1 as we can use Philip Hall's inequality that $\operatorname{def}(G) \leq \beta_{1}(G)-d\left(H_{2}(G ; \mathbb{Z})\right.$ ) (see [64], 14.1.5). We have $H_{n}(T ; \mathbb{Z})=\mathbb{Z} \times \mathbb{Z}$ for all $n \geq 1$ by [21], Theorem 7.1 , so that by using the Meier-Vietoris sequence for a free product we get $\beta_{1}(G)=3$ and $H_{2}(G ; \mathbb{Z})=\mathbb{Z} \times \mathbb{Z}$.

Therefore any formulation of a possible Tits alternative for subgroups of deficiency 1 groups will need to avoid freely decomposable examples.

\section{2-generator 1-relator groups of height 1}

So far our attempts to prove that various deficiency 1 groups are large have needed some hypothesis on the group, such as being free-by-cyclic or LERF. In this section we concentrate on 2-generator 1-relator groups and look for conditions where we can conclude that such a group is large using information obtained directly from a given presentation. We fall into two very different cases: a group $G$ of the form $\langle x, y \mid r(x, y)\rangle$ has $\beta_{1}(G)$ equal to either 1 or 2 , the latter occurring exactly when $r \in F_{2}^{\prime}$. First let us concentrate on the former case. There is an automorphism $\alpha$ of $F_{2}$, with let us say $\alpha(x)=a$ and $\alpha(y)=t$, such that $r\left(\alpha^{-1}(a), \alpha^{-1}(t)\right)$ has exponent sum 0 in $t$ when written as a word $w$ in $a$ and $t$. This is a consequence of the fact that the kernel of the natural map from $\operatorname{Aut}\left(F_{2}\right)$ to $\operatorname{Aut}\left(F_{2} / F_{2}^{\prime}\right) \cong G L(2, \mathbb{Z})$ is the group of inner automorphisms of $F_{2}$. Thus by making a change of free basis for the group $F_{2}$ we can assume that the presentation is of the form $\langle a, t \mid w(a, t)\rangle$ where the word $w$ has exponent sum 0 in $t$ and is cyclically reduced. Consequently we can define the height of such words: we can rewrite $w$ as a word in $a_{i}=t^{i} a t^{-i}$ for $i \in \mathbb{Z}$ to obtain the new word $v$, but only finitely many letters $a_{i}$ will actually appear in $v$ (this is sometimes referred to as Magnus rewriting). If $a_{m}$ is the smallest and $a_{M}$ the largest letter to appear in $v$ then the height of $w$ is defined to be $M-m \geq 0$, which is invariant under cyclic permutations and taking inverses.

If the height is zero then we can only have $w=a^{i}$, so $G$ is large (or $\mathbb{Z}$ for $|i|=1$ ). We now consider height one words where without loss of generality we can assume that

$$
w=t a^{i_{1}} t^{-1} a^{i_{2}} \ldots t a^{i_{2 k-1}} t^{-1} a^{i_{2 k}}, \text { so } v=a_{1}^{i_{1}} a_{0}^{i_{2}} \ldots a_{1}^{i_{2 k-1}} a_{0}^{i_{2 k}} .
$$

Note that for $\chi(t)=1, \chi(a)=0$ we have $\Delta_{G, \chi}(t)=\left(i_{1}+\cdots+i_{2 k-1}\right) t+i_{2}+\cdots+i_{2 k}$ and for $\beta_{1}(G)=1$ this is the Alexander polynomial $\Delta_{G}$.

If $\beta_{1}(G)=2$ then the exponent sum of $x$ and $y$ is zero, so we can talk about the height of either letter. Moreover a change of basis will preserve this property but could well vary the heights. The results that follow are also satisfied by groups $G$ with $\beta_{1}(G)=2$, where we interpret a height 1 word as there exists a free basis where the word has height 1 with respect to one of the letters. Given a finitely generated group $G$, the finite residual $R_{G}$ is the intersection of all finite index subgroups of $G$. Consequently it is the trivial subgroup if and only if $G$ is residually finite. 
Theorem 4.1. If $G=\langle a, t \mid w\rangle$ where $w$ is a height 1 word then either $G$ is large or the finite residual $R_{G}=G^{\prime \prime}$, in which case $G / R_{G}$ is metabelian and all finite images of $G$ are metacyclic.

Proof. By writing $w$ in terms of $a_{0}, a_{1}$ to get the word $v$ as in (2), we see that $G$ is an HNN extension of the 1-relator group $H=\left\langle a_{0}, a_{1} \mid v\left(a_{0}, a_{1}\right)\right\rangle$ with associated cyclic subgroups $A_{0}=\left\langle a_{0}\right\rangle$ and $A_{1}=\left\langle a_{1}\right\rangle$. We can now use Lubotzky's result as mentioned in the previous section: although it appears to require that $G$ is LERF, all we need to conclude largeness for $G$ is the existence of a homomorphism $\phi$ of $G$ onto a finite group with $\phi(H) \neq \phi\left(A_{0}\right)$. Let us suppose that no such homomorphism exists and let $\theta$ be any homomorphism from $G$ to a finite group. As $\theta(H)=\theta\left(A_{0}\right)$ which is cyclic and $t$ conjugates $a_{0}$ into $H$, we have that $\theta(H)$ is normal in $\theta(G)$ and cyclic, with $\theta(G) / \theta(H) \cong\langle\theta(t)\rangle$ cyclic as well. This means that all finite images of $G$ are metacyclic and if $x \in G^{\prime \prime}$ but $x \notin R_{G}$, we would take a homomorphism $\theta$ from $G$ onto a finite group $F$ with $\theta(x) \neq e$ so that $F^{\prime \prime}$ is non-trivial, but this is a contradiction. Consequently $G^{\prime \prime} \leq R_{G}$, but Philip Hall's result that a finitely generated metabelian group is residually finite implies that $R_{G} \leq G^{\prime \prime}$.

Suppose $G$ in Theorem 4.1 is not large, so that $G / R_{G}$ is metabelian. It is also the case that the normal closure of the element $a$ in $G / R_{G}$, which is generated by elements of the form $t^{i} a t^{-i}$ for $i \in \mathbb{Z}$, is not just metabelian but abelian. This is because if there existed $\left[t^{i} a t^{-i}, a\right] \notin R_{G}$ then we could take a finite image of $G$ in which this was also non-trivial, but this contradicts the fact that the cyclic group $\langle a\rangle$ is normal in every finite image. We will use this fact in the following discussion of height 1 groups which are not large.

This gives us a strict dichotomy in the behaviour of height 1 presentations. However the result does not tell us how to work out in which category a given presentation falls. Luckily there is a straightforward way of determining largeness once a major result of Zelmanov is used.

Corollary 4.2. If $G=\langle a, t \mid w\rangle$ where $w$ is a height 1 word then $G$ is large if and only if there exists $H \leq_{f} G$ with $d\left(H / H^{\prime}\right) \geq 3$, where $d$ is the minimum number of generators of a finitely generated group.

Proof. The only if direction is clear. [68] states that if we have a finite presentation $P$ of a group $G$ with $\operatorname{def}(P)+d^{2} / 4-d>0$ where $d=d\left(G / G^{\prime}\right)$ is at least 2 then there exists a prime $p$ such that the pro- $p$ completion of $G$ contains a non-abelian free pro- $p$ group. In our case the deficiency is 1 , so this is true if $d \geq 3$. But if $G^{\prime \prime}=R_{G}$ then all finite images $F$ of $G$ have $F^{\prime \prime}$ trivial, so this is also true for the profinite or pro- $p$ completion of $G$. Consequently any pro- $p$ completion is soluble and cannot contain a non-abelian free group.

However $G$ is 2-generated, so we cannot have $d\left(G / G^{\prime}\right) \geq 3$ in any case. But for $H \leq_{f} G$ we still have deficiency 1 , with $H^{\prime \prime} \leq G^{\prime \prime}$ and $R_{G}=R_{H}$, so if $G^{\prime \prime}=R_{G}$ and $d\left(H / H^{\prime}\right) \geq 3$ we have a contradiction. 
We now give a summary of height 1 group presentations that have appeared in the literature. If we define the length of height 1 words to be $k$ in (2) then it is clear that length 1 words are just the standard presentations of the Baumslag-Solitar groups $\mathrm{BS}(m, n)$. In [4] Baumslag introduced a family $C(m, n)$ of groups with presentations

$$
\left.\langle a, t|\left(\text { tat }^{-1}\right) a^{m}\left(t a t^{-1}\right)^{-1}=a^{n}\right\rangle
$$

which generalises the group $C(1,2)$ that first appeared in [2] with the comment that they are 1-relator groups which are as far from being residually finite as possible. For our purposes we will interpret a finitely generated group $G$ as being "as far from being residually finite as possible" in three ways: the first is that $G$ (is infinite but) has no proper finite index subgroups whatsoever, or equivalently $G=R_{G}$. However this will never arise with deficiency 1 groups as $G / G^{\prime}$ is infinite.

The next strictest interpretation is that ( $G$ is non-abelian but) $G^{\prime}=R_{G}$, or equivalently every finite image is abelian. To be succinct, we will say that $G$ is proabelian because this is equivalent to the profinite completion of $G$ being proabelian (or abelian). This property of finitely generated groups was examined in [27]. A finite index subgroup of a proabelian group is also proabelian and a finitely presented proabelian group $G$ (with $\beta_{1}(G)>0$ ) has $\Delta_{G}=1$ (which can be seen because the Alexander polynomial of $G$ is the same as that of $G / R_{G}$ and this will be 1 for finitely generated infinite abelian groups). If $G$ is a deficiency 1 group with the abelianisation $G / G^{\prime}$ equal to $\mathbb{Z} \times T$ then $G$ being proabelian and $\beta_{1}(G)=1$ implies that $T$ must be trivial because here $\left|\Delta_{G}(1)\right|=|T|$. We cannot have $d\left(G / G^{\prime}\right) \geq 3$ from above, so that if $G$ is proabelian and $\beta_{1}(G) \geq 2$ then $G / G^{\prime}=\mathbb{Z} \times \mathbb{Z}$. Moreover if $H / H^{\prime}=\mathbb{Z}$ for all $H \leq_{f} G$ (or $H / H^{\prime}$ is $\mathbb{Z} \times \mathbb{Z}$ throughout) then $G$ is proabelian by [27] Proposition 3.4. In particular if $G$ has deficiency 1 with $\beta_{1}(G)=2$ then either $G$ is proabelian (so that $G / R_{G}=\mathbb{Z} \times \mathbb{Z}$ ) or a pro- $p$ completion of $G$ contains a non-abelian free pro- $p$ group (so that $G / R_{G}$ is "big").

The first example of a proabelian finitely generated group that is given by a 1-relator presentation is $C(1,2)$; indeed every finite image is cyclic as the abelianisation of $C(1,2)$ is $\mathbb{Z}$.

Finally we could also look at when ( $G$ is not metabelian but) $G^{\prime \prime}=R_{G}$, or equivalently every finite image is metabelian, for which we will similarly write that $G$ is prometabelian. As we have $R_{G} \leq G^{\prime \prime}$ for any finitely generated group (although not necessarily $R_{G} \leq G^{\prime \prime \prime}$ ) this is also a natural concept, with a prometabelian group $G$ being proabelian if and only if $G^{\prime}=G^{\prime \prime}$. Moreover it is also fair to say that finitely generated proabelian and prometabelian groups are "as far from being large as possible".

If we have a height 1 group $G=\langle a, t \mid w(a, t)\rangle=\left\langle a_{0}, a_{1}, t\right| t a_{0} t^{-1}=$ $\left.a_{1}, v\left(a_{0}, a_{1}\right)\right\rangle$ which is not large then by the comment after Theorem 4.1 we have that $a_{0}$ commutes with $a_{1}$ in $G / R_{G}$. In particular if the exponent sum of $a_{0}$ in $v\left(a_{0}, a_{1}\right)$ is $d$ and that of $a_{1}$ is $c$ then $G / G^{\prime \prime}$ has the presentation

$$
\left.\langle a, t| t a^{c} t^{-1}=a^{-d},\left[t^{i} a t^{-i}, a\right] \text { for } i \in \mathbb{Z}\right\rangle,
$$


which is the same as for $\operatorname{BS}(c,-d) / \mathrm{BS}(c,-d)^{\prime \prime}$. Thus any result which depends purely on the finite images of $\operatorname{BS}(m, n)$ for $m$ and $n$ coprime applies identically for any non-large group $G$ of height 1 (and if $c$ and $d$ are not coprime then $G$ is large anyway). In particular the formulae for the number of finite index subgroups of $\mathrm{BS}(m, n)$ in [37] and the number of finite index normal subgroups in [26] apply equally for $G$. However there is a potential problem here: we do not know whether these groups are merely nonstandard presentations of $\mathrm{BS}(m, n)$. For instance on putting $b=a^{2}$ and substituting in for the standard presentation of $\mathrm{BS}(2,3)$ we have $a=t b t^{-1} b^{-1}$ and so we obtain an alternative height 1 presentation

$$
\left\langle b, t \mid t b t^{-1} b^{-1} t b t^{-1} b^{-2}\right\rangle .
$$

The problem is a lack of invariants which are able to distinguish that two given presentations do not define isomorphic groups. We see in these cases that any such invariant which is calculated using information obtained solely from the finite images of a group is here doomed to failure.

As for 1-relator proabelian groups that are not abelian, we can only have presentations with 2 generators. We have already mentioned $C(1,2)$ above and in [33] the example $C(2,3)$ was introduced where it was shown that for all $H \leq_{f} C(2,3)$ we have $H / H^{\prime}=\mathbb{Z}$, so $C(2,3)$ is proabelian by [27], Proposition 3.4. Moreover if we regard $C(2,3)$ as an HNN extension of BS $(2,3)$ by adding the stable letter $s$ with $s^{-1}=t$ then we can iterate this process indefinitely and keep on obtaining new 1-relator height 1 group presentations. This HNN extension when applied to $C(1,2)$ was also used in [28] to give an example of a higher dimensional knot whose infinite cyclic cover is not simply connected but which has no proper finite covers. The group $C(1,2)$ (which is sometimes referred to as the Baumslag-Gersten group) also appears in [38] where it is shown that its Dehn function grows faster than every iterated exponential, and again in [22] where it is shown that it is isomorphic to

$$
\left\langle a, t \mid\left(t a^{2^{k}} t^{-1}\right) a\left(t a^{2^{k}} t^{-1}\right)^{-1}=a^{2}\right\rangle
$$

for any $k \geq 0$. The paper [23] considers a more general family $D(k, m, n)$ containing the groups $C(m, n)$ where the conjugating element $t a t^{-1}$ can be $t a^{k} t^{-1}$. These were used in [17] to give a pair of non-isomorphic 1-relator groups, each of which is a homomorphic image of the other. Also the examples

$$
\left\langle a, t \mid\left(t^{k} a t^{-k}\right) a\left(t^{k} a t^{-k}\right)^{-1}=a^{2}\right\rangle
$$

of proabelian groups for $k \geq 1$ are mentioned in [57]: note this implies that a proabelian 1-relator group can have arbitrary height.

We can incorporate all of these examples in the next result. Although the proof is really that of Higman in [40] when giving the first example of an infinite finitely generated simple group, the aim here is to express it in as general terms as possible. A very similar interpretation of Higman's result is Lemma 2 in [6]. 
Theorem 4.3. Suppose that $g$ and $h$ are elements of a finite group $F$ with $h^{k} g^{m} h^{-k}=$ $g^{n}$ for integers $k, m, n$ where $|m-n|=1$. Suppose further that $g$ and $h$ have equal orders. Then this order is always 1.

Proof. If $r$ is the order of $g$ and $h$ then by considering $h^{r k} g^{m^{r}} h^{-r k}$ which is equal to $g^{n^{r}}$, we must have $r$ dividing $m^{r}-n^{r}$. So if $r \neq 1$ and $p$ is the smallest prime dividing $r$, we have $\left(m n^{-1}\right)^{r} \equiv 1 \bmod p$ where we can assume without loss of generality that $n$ and $p$ are coprime. Thus the order of $m n^{-1}$ divides $r$ and $p-1$ so $p$ divides $m-n= \pm 1$.

Corollary 4.4. Suppose that $H=\langle a, t \mid w(a, t)\rangle$ where $w$ has exponent sum 0 in $t$ and is of height 1 . Then the HNN extension $G=\langle H, s\rangle$, where sas ${ }^{-1}=t$, is large if $H / H^{\prime} \neq \mathbb{Z}$ and proabelian if $H / H^{\prime}=\mathbb{Z}$, but $H$ is not large.

Proof. $G=\left\langle a, s \mid w\left(a, s^{-1}\right)\right\rangle$ is also a 1-relator group of height 1 and if the Alexander polynomial of $H$ (with respect to the homomorphism $\chi(t)=1, \chi(a)=0$ ) is $f(t)=c t+d$ then that for $G$ is $c+d=f(1)$. However as $H$ is a group with a deficiency 1 presentation, we have that $|f(1)|$ is the order of $T$, where $H / H^{\prime}=\mathbb{Z} \times T$. Consequently if $T$ is non-trivial then we can take a prime $p$ dividing the order of $T$. We then use the fact that as $G$ also has a presentation of deficiency 1 , the $\bmod p$ Alexander polynomial for $G$ is just the Alexander polynomial for $G$ reduced $\bmod p$ and so is 0 here. Thus $G$ is large by Howie's result.

Now suppose that $|T|=1$ and $H$ is not large. Let $\theta$ be any homomorphism from $G$ to a finite group $F$. We have that in $F$ the relation $t a^{c} t^{-1}=a^{-d}$ holds because $\theta(H)$ is a finite image of $H$ and $H^{\prime \prime}=R_{H}$. But $t$ and $a$ are conjugate in $F$ via the image of $s$, so we can use Theorem 4.3 to conclude that $\theta(t)=\theta(a)=e$. Thus the image of $G$ in $F$ is generated by $\theta(s)$.

Note that this means the group $C(m, n)$ above is not large if and only if $|m-n|=1$. This is in disagreement with [4], Theorem 4, where Part 2 states (but proofs are not given) that $C(m, n)$ is proabelian if $m$ and $n$ are distinct primes. However in Section 3 of that paper it is mentioned that if $m=n+1$ then $C(m, n)$ is proabelian, so we believe that is what was meant in the theorem.

Corollary 4.2 allows us, with the help of a computer, to gather statistics on what proportions of height 1 groups $G$ are large by searching through finite index subgroups $H$ of $G$ until $d\left(H / H^{\prime}\right) \geq 3$, or until we give up on this method and begin to suspect that $G$ might not be large. This is done in [29], Section 3.2, where we looked at all words $w(a, t)$ with exponent sum zero in $t$ and of height 1 , up to word length 14 . We found that all words give rise to large groups, apart from Baumslag-Solitar groups with coprime parameters along with a table of about 20 entries. Each of these is either a Baumslag-Solitar group (but not given by the standard presentation) or one from the family $D(k, m, n)$, with the possible exception of two words of length 13 , where we could not identify the group nor determine largeness. We note here in relation to 
Corollary 4.4 that the computer tells us that the group $\left\langle a, t \mid t a^{2} t^{-1} a^{-1} t a^{-1} t^{-1} a^{-1}\right\rangle$ is large but has Alexander polynomial $t-2$, which is the same as for BS(1,2), and forming the $\mathrm{HNN}$ extension of this group using $\mathrm{sas}^{-1}=t$ results in a height 1 group which is large but with Alexander polynomial equal to 1 .

Corollary 4.5. If $G_{0}=\langle a, t \mid w\rangle$ is proabelian then $G_{1}=\left\langle a, t \mid v^{k} w^{m} v^{-k}=w^{n}\right\rangle$ is proabelian, where $v$ is a conjugate element of $w$ in $F_{2}$ and $|m-n|=1$.

Proof. Given any finite image $F$ of $G_{1}$, we set $h$ equal to the image of $v$ and $g$ equal to the image of $w$ and apply Theorem 4.3. We conclude that the image of $w$ is trivial in $F$, so $F$ is a finite image of $G_{0}$ and hence is abelian.

Thus we see how to create lots of proabelian 1-relator groups by starting with $w=a$ (or indeed $w$ equal to any element of a free basis for $F_{2}$ ) and then iterating in Corollary 4.5. The same construction is given in [6], Theorem 1, where it is shown that for a 1-relator group $G=\langle a, b, \ldots \mid w\rangle$, if $v=u w u^{-1}$ where $u$ does not commute with $w$ in the ambient free group then the group $\bar{G}=\left\langle a, b, \ldots \mid v w v^{-1}=w^{2}\right\rangle$ has the same finite images as $G$, but $w \neq 1$ in $\bar{G}$. Therefore $\bar{G}$ is not residually finite, thus if $G$ is then $\bar{G} \not G$, but $\bar{G}$ surjects onto $G$. This allows us to construct many 2-generator 1-relator groups $\bar{G}$ which are large but not residually finite, by inputting any 2-generator 1-relator group $G$ which is large so that $\bar{G}$ will be too.

This construction also allows us to give an example of a 1-relator group $G$ with $\beta_{1}(G)=2$ that is proabelian, the existence of which was not previously known. By putting in $w=\left[t, a^{-1}\right]$ and $u=a$ so that $G=\mathbb{Z} \times \mathbb{Z}$ above, we see that the group

$$
\bar{G}=\left\langle a, t \mid[a, t]\left[t, a^{-1}\right][a, t]^{-1}=\left[t, a^{-1}\right]^{2}\right\rangle
$$

which is Corollary 2 in [6] is proabelian (hence not large) with $\beta_{1}(G)=2$ but is not residually finite, hence not equal to $\mathbb{Z} \times \mathbb{Z}$.

We note that the relation above is of height 1 with respect to $t$. However there are also groups with first Betti number 2 and height 1 which we can prove are large:

Corollary 4.6. If $G=\langle a, t \mid w(a, t)\rangle$ where $\beta_{1}(G)=2$ and $w$ is a height 1 word of the form in (2) then $G$ is large if $\left|i_{1}+i_{3}+\cdots+i_{2 k-1}\right| \neq \pm 1$. Otherwise $G$ is either proabelian, equal to $\mathbb{Z} \times \mathbb{Z}$ or large.

Proof. We have that $\beta_{1}(G)=2$ implies $i_{1}+i_{2}+\cdots+i_{2 k}=0$ and so $\Delta_{G, \chi}(t)=$ $n(t-1)$ where $n=i_{1}+i_{3}+\cdots+i_{2 k-1}$ with $\chi(a)=0, \chi(t)=1$. Thus if $|n| \neq \pm 1$ we have largeness because $\Delta_{G, \chi}^{p} \equiv 0$ modulo a suitable prime $p$. We also have largeness by Corollary 4.2 unless $H / H^{\prime}=\mathbb{Z} \times \mathbb{Z}$ for all $H \leq_{f} G$. If this is so then [27], Proposition 3.4, shows that if $G$ is quotiented out by its finite residual $R_{G}$ then we have $G / R_{G} \cong \mathbb{Z} \times \mathbb{Z}$. In particular $G$ is proabelian if $R_{G}$ is non-trivial and equal to $\mathbb{Z} \times \mathbb{Z}$ otherwise. Moreover it is known by [54], Section 4.4, that a 2-generator 1-relator group $\langle a, t \mid r\rangle$ is equal to $\mathbb{Z} \times \mathbb{Z}$ if and only if $r=[a, t]^{ \pm 1}$ 
or a cyclic conjugate when $r$ is cyclically reduced, so we only have $G=\mathbb{Z} \times \mathbb{Z}$ if $w=t a^{ \pm 1} t^{-1} a^{\mp 1}$.

For instance the group $\left\langle a, b, t \mid a b a^{2}=b^{2}, t a^{3} t^{-1}=b\right\rangle$ is shown in [18], Proposition 19, to be word hyperbolic and of the form $F_{6} \rtimes_{\alpha} \mathbb{Z}$. As such groups are residually finite and eliminating $b$ gives a height 1 word with respect to $t$, we can use Corollary 4.6 to obtain another group satisfying the conditions of Corollary 2.4 without using the computer.

\section{Problem list}

Here we list some problems in Group Theory with particular emphasis on finitely generated and finitely presented groups. The aim is to put together questions which may well have appeared somewhere in print but which are not to be found in the standard problem lists (for instance the Kourovka notebook [48], the New York list [59] and Mladen Bestvina's questions in Geometric Group Theory [8]). Also there is an emphasis on questions which can be stated using only group theoretic concepts, although it may well be that solutions require topological, geometric or other techniques. In some cases the credits refer to those from whom we first heard about the question; we apologise if this is not the original source. The order of appearance places what should be the most general questions first and then specialises until we reach the topics of this paper.

The list was first compiled in March 2008 and we have added updates where known in January 2010.

\section{Finiteness questions}

1 (J. S. Wilson [67]). Is there a finitely presented and residually finite group which is neither virtually soluble nor contains a non-abelian free group?

Notes. If we replace finitely presented with finitely generated then certainly a range of examples are known. If however we keep finitely presented and remove residually finite then the list becomes much shorter. We know of only four constructions: Thompson's group $F$, the Houghton groups as in [43], the finitely presented Grigorchuk group (which is an ascending HNN extension of the well-known Grigorchuk group) and the non-amenable monsters (which are ascending HNN extensions of finitely generated infinite groups with finite exponent) in [63]. All of these are far from being residually finite. Of course if we weaken finitely presented to finitely generated but strengthen residually finite to linear then there are no examples by the Tits alternative.

A variation might be to strengthen the conditions in other ways (e.g. LERF, coherent, of type FP ) with the aim of proving such an example does not exist. 
Update. No progress known, even with the strengthened conditions. We remark though that a proof of the amenability of Thompson's group $F$ has been put forward by E. T. Shavgulidze although this has been disputed.

The Prüfer rank of a group is the supremum of the minimum number of generators over all finitely generated subgroups.

2. If a group has infinite Prüfer rank then must it have an infinitely generated subgroup?

Notes. This question has interesting implications either way. We say that a group has max (or is Noetherian) if it and every subgroup is finitely generated. The question of whether a group with max is virtually polycyclic was settled in the negative by Olshankskii's construction of Tarski monsters, but these have finite Prüfer rank. Moreover this property (like max) is preserved by subgroups, quotients and extensions. Thus a counterexample in Question 2 would be a genuinely new example of a group with max as it would not be obtained from known examples by these operations.

If however the answer is yes then a result of Lubotzky and Mann in [52] says that a finitely generated, residually finite group with finite Prüfer rank is virtually soluble. Thus a residually finite group with max would be virtually polycyclic, giving a positive answer to Question 31 in the first Kourovka notebook, credited to M. I. Kargapolov.

Update. The answer is no, as pointed out independently by A. Minasyan and A. Klyachko using the same method: Theorem 35.1 of [61] shows that for any countable family of countable groups, there exists a 2-generated simple group $G$ such that every proper subgroup of $G$ is either cyclic or contained in a conjugate of a member of this family. Thus we can take our family to be $\mathbb{Z}^{n}$ for $n \in \mathbb{N}$. However a residually finite example is still unknown.

3. If $G / N$ and $N$ are both virtually soluble then is $G$ ?

Notes. This is true and straightforward with virtually soluble replaced by soluble.

Update. This is true, as pointed out independently by P. Linnell, by A. Minasyan and by A. Klyachko. We outline the argument: let $M$ be the unique maximal normal subgroup of $N$ (which exists as the product of two soluble normal subgroups is itself soluble and normal). Then $M$ is characteristic in $N$ so normal in $G$. Thus, by replacing $G$ and $N$ with $G / M$ and $N / M$ respectively, we can assume that $N$ is finite with no non-trivial soluble normal subgroups. The centraliser $C$ of $N$ in $G$ has finite index in $G$ and $C \cap N$ is abelian thus trivial. Now we are done because $C$ is isomorphic to the virtually soluble group $C N / N$.

This is taken further in [46] Section 5: let the virtual derived length $\operatorname{vdl}(G)$ of a virtually soluble group $G$ be the smallest derived length of a finite index subgroup. There it is shown that $\operatorname{vdl}(G) \leq \operatorname{vdl}(G / N)+\operatorname{vdl}(N)+1$ and this is best possible.

4. Is there a finitely presented group that is elementary amenable but not virtually soluble? 
Notes. In [41] Hillman gives an infinitely generated example and then uses it to obtain a finitely generated example.

Update. P. Linnell points out that this is now in the Kourovka notebook as Problem 16.52 and Y. de Cornulier mentions the Houghton groups as examples.

\section{Amenability and properties $(T)$ and $(\tau)$}

5. Do all finitely generated (or finitely presented) infinite amenable groups have a proper finite index subgroup?

Notes. The rationals show this is not true for infinitely generated groups. We have the dichotomy that no infinite discrete group can have property $(T)$ and be amenable. For property $(\tau)$ (always assumed here to be with respect to all normal finite index subgroups) we have by [50], Proposition 3.3.7, that a finitely generated amenable group with infinitely many finite index subgroups does not have $(\tau)$, but groups with finitely many finite index subgroups trivially have property $(\tau)$. If the answer is yes then we regain our dichotomy for finitely generated/presented groups.

An old question asks if there is a finitely generated infinite simple group which is amenable. This is equivalent to having a finitely generated infinite amenable group with no proper finite index subgroups, as we can quotient out by a maximal normal subgroup. Infinitely generated simple amenable groups certainly exist, for instance the union $\bigcup A_{n}$ of alternating groups. It has also been asked whether there are finitely presented infinite simple amenable groups, but those wishing to establish their existence would need to answer the next question in the negative.

6. Does an infinite finitely presented simple group necessarily contain a non-abelian free group?

Notes. This is not true for infinite finitely generated simple groups, but for all known finitely presented examples the answer seems to be yes.

7 (Lackenby). If a finitely presented group has zero virtual first Betti number then must it have property $(\tau)$ ?

Notes. The Grigorchuk group shows that this is not true for finitely generated groups as it is amenable with infinitely many finite index subgroups.

We recall that property $(T)$ implies finite generation as well as implying property $(\tau)$, which in turn implies zero virtual first Betti number.

\section{Subgroup separability}

8 (J. S. Wilson). Is there a finitely generated group which is not virtually polycyclic but where every subgroup is the intersection of finite index subgroups? 
Notes. The paper [45] shows that there are no examples when the group is hyper(abelian or finite) which covers being virtually soluble. As for a finitely presented example, this would be residually finite and would not contain $F_{2}$, so would provide a yes answer to Question 1.

9 (Long and Reid [49], Question 4.5). Is there a finitely generated infinite group which is LERF and has property $(T)$ ?

10. Is there a finitely presented infinite group which is LERF and which has zero virtual first Betti number?

Notes. A finitely presented example for Question 9 (or just one with LERF and property $(\tau)$ ) would of course answer this. However the Grigorchuk group is a finitely generated infinite LERF group which has zero virtual first Betti number.

\section{Largeness}

11. If a finitely generated or finitely presented group has the biggest possible subgroup growth, that is of strict type $n^{n}$, then is it large?

Update. No progress known, but J. -C. Schlage-Puchta has looked at this and conjectures that there should exist finitely generated torsion groups with this type of subgroup growth.

12. Is there an algorithm to determine whether or not the group given by a finite presentation has a proper finite index subgroup?

Notes. It is unknown whether there is an algorithm to detect largeness in finitely presented groups. There are partial algorithms which will terminate with the answer yes if a finitely presented group is large and which may return no or not terminate otherwise, see for instance [29]. If we have a complete algorithm for largeness then, as pointed out by D. Groves and I. Leary, we have yes to this question because a group $G$ has proper finite index subgroups if and only if $G * G * G$ is large.

13. If a finitely presented group has infinite virtual first Betti number then must it be large? Does it contain $F_{2}$ ? Must it be not virtually soluble?

Notes. These seem interesting questions. The first point to make is that these are all false for finitely generated groups, as in [32] it is pointed out that the soluble and $\mathbb{R}$-linear group $\mathbb{Z} \imath \mathbb{Z}$ has infinite virtual first Betti number. Therefore one would expect to find counterexamples by taking a group surjecting to $\mathbb{Z} \imath \mathbb{Z}$. But Baumslag shows in [3], Chapter IV, Theorem 7, that a finitely presented group surjecting to $\mathbb{Z} \imath \mathbb{Z}$ is large. 
Update. All three questions are still open, but here is an intriguing observation for the large case: it is enough to restrict to torsion-free word hyperbolic groups. This is because if $P$ is a finitely presented group then by [60], building on a construction of Rips, there exists a torsion-free word hyperbolic group $G$ such that $G / N \cong P$ and $N$ has property (T). If $P$ has infinite virtual first Betti number then so does $G$. However $P$ and $N$ both being finitely generated but not large implies that $G$ is not large.

We can also ask about growth of first Betti numbers in finite covers.

14. If a finitely presented group $G$ has a sequence of finite index subgroups $H_{n}$ such that $\beta_{1}\left(H_{n}\right) /\left[G: H_{n}\right] \geq c>0$ for all $n$ then is $G$ large?

Notes. If $H_{n} \unlhd G$ with the sequence nested and such that $\bigcap H_{n}$ is trivial (thus implying that $G$ is residually finite) then this limit exists and is the first $L^{2}$-Betti number by Lück's Approximation Theorem. However groups of the form $F_{n} \rtimes_{\alpha} \mathbb{Z}$ have zero first $L^{2}$-Betti number, but any large example will have a sequence as in the question.

If the sequence $\left(H_{n}\right)$ above is such that there is a surjective homomorphism $\chi: G \rightarrow \mathbb{Z}$ with $K=\operatorname{ker} \chi \leq H_{n}$ for all $n$ then $G$ is large. Indeed we only require that $\beta_{1}\left(H_{n}\right)$ is unbounded, or even $\beta_{1}\left(H_{n} ; \mathbb{Z} / p \mathbb{Z}\right)$ for some prime $p$. This is because

$$
\beta_{1}\left(H_{n} ; \mathbb{F}\right) \leq \beta_{1}(K ; \mathbb{F})+1 \text { for } \mathbb{F}=\mathbb{Q} \text { or } \mathbb{Z} / p \mathbb{Z},
$$

so if we have an unbounded sequence then $\Delta_{G, \chi}^{\mathbb{F}}$ is zero, with Howie's result implying that for all large $k$ the subgroup $K G^{k}$ has a surjection onto $C_{p} * C_{p} * C_{p}$ (or $\mathbb{Z} * \mathbb{Z} * \mathbb{Z}$ for $\mathbb{F}=\mathbb{Q}$ ). However the counterexample $\mathbb{Z} \imath \mathbb{Z}$ above for finitely generated groups also has the first Betti number growing linearly in a sequence of subgroups containing ker $\chi$.

One could also formulate variations of Question 14 using first Betti numbers modulo a prime $p$. However Question 13 is no longer true in this more general setting: consider the $\mathbb{R}$-linear Baumslag-Remeslennikov soluble group

$$
G=\left\langle a, s, t \mid t^{-1} t^{-1}=a_{s a s^{-1}},\left[a, s a s^{-1}\right]=1=[s, t]\right\rangle .
$$

By using the binomial theorem modulo any prime $p$, it is not hard to show that we have subgroups $H_{n}$ with $\beta_{1}\left(H_{n} ; \mathbb{Z} / p \mathbb{Z}\right) \geq 2+2 p^{n-1}$ where $G^{\prime} \unlhd H_{n} \unlhd G$ is the finite abelian cover corresponding to

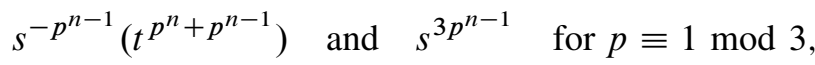

$$
\begin{aligned}
& t^{p^{n}+p^{n-1}} \text { and } s^{3 p^{n-1}} \text { for } p \equiv 2 \bmod 3 \text {, } \\
& t^{p^{n}+p^{n-1}} \text { and } s^{8 p^{n-1}} \text { for } p=3 \text {. }
\end{aligned}
$$

This is the fastest possible growth of $\beta_{1}(H ; \mathbb{Z} / p \mathbb{Z})$ because Corollary 1.4 in [67] states that if $G$ is finitely presented and soluble then there exists $\kappa>0$ such that $d\left(H / H^{\prime}\right) \leq \kappa|G: H|^{1 / 2}$ for all $H \leq_{f} G$. 
15. Fix an integer $k \leq 1$. Is there $f(k)$ such that whenever a group $G$ has a presentation of deficiency $k$ and $\beta_{1}(G)>f(k)$ then $G$ is large?

This seems less and less likely as $k$ gets smaller. It is of course true for $k \geq 2$.

16 (J. S. Wilson [67]). If a group $G$ has a finite presentation $P$ such that $\operatorname{def}(P)+d^{2} / 4-d>0$ where $d \geq 2$ is the minimum number of generators of $G / G^{\prime}$ then does $G$ contain a non-abelian free group?

Notes. Bartholdi has recently proved in [1] that Golod-Shafarevich groups are not amenable. These are groups with a presentation (of finitely many generators but possibly infinitely many relators) possessing a certain condition which is certainly satisfied by finite presentations of the form above. This can be seen as providing evidence for the answer yes because a counterexample would have the extremely rare property of being a finitely presented non-amenable group without $F_{2}$ as a subgroup. Lackenby and Lubotzky ask for a group with a finite presentation as above which is not large: surely these exist? There is also the question (credited to Lubotzky and Zelmanov) of whether a group with such a presentation can have property $(T)$ or $(\tau)$. Recently Ershov in [34] has constructed Golod-Shafarevich groups with property $(T)$.

Update. Bartholdi's paper is regarded by the author as having a gap. However since then a major result in [35] is that any Golod-Shafarevich group has an infinite quotient with property $(T)$, thus is non amenable (indeed it is shown that these groups are uniformly non amenable).

\section{Deficiency 1 groups}

17. Is every deficiency 1 group that does not contain $F_{2}$ isomorphic to $\mathrm{BS}(1, m)$ ?

Notes. For evidence, see Section 3.

18. Is every residually finite deficiency 1 group that is not large isomorphic to $\mathrm{BS}(1, m)$ ?

Notes. In particular are all word hyperbolic groups of the form $F_{n} \rtimes_{\alpha} \mathbb{Z}$ for $n \geq 2$ large? A proof could be attempted in two parts: assume the virtual first Betti number is at least 2 to obtain largeness, and separately establish this assumption which is Question 12.16 in [8] due to Casson.

19. If a deficiency 1 group $G$ has (virtual) first Betti number at least 3 then is it large? If the minimum number of generators of $G / G^{\prime}$ (or $H / H^{\prime}$ for $H \leq_{f} G$ ) is at least 3 then is $G$ large?

Notes. The first part is Question 15 for $k=1$ and $f(1)=2$, which is certainly more believable here. The example before Corollary 4.6 shows that we cannot replace "(virtual) first Betti number at least 3" with "(virtual) first Betti number at least 2 and $G \neq \mathbb{Z} \times \mathbb{Z}$ (or BS $(1,-1)$ )", even in the 1-relator case. 
2-generator 1-relator presentations. In this special case Question 17 is true, but Questions 18 and 19 are unknown.

Update. Major computation was undertaken in [29], which provides evidence in support of both questions. In particular they are both true for all such presentations where the relator has length at most 12 and zero exponent sum in at least one generator, as well as length at most 18 and zero exponent sum in both generators.

20. Let $G$ be a group with a 2-generator 1-relator presentation where the relator is not a proper power. Suppose that $G$ is residually finite, then does $G$ have a finite index subgroup $H$ which is an ascending $\mathrm{HNN}$ extension of a finitely generated free group?

Notes. If so then every finite index subgroup of $H$, and $G$, has deficiency 1 . However if the relator is a proper power, which is exactly when $G$ has torsion, then often $G$ has a finite index subgroup of deficiency at least 2 and in any case $G$ is known to be large (although [4], Problem 4, asks if a 1-relator group with torsion is virtually free-by-cyclic: here the free part could be infinitely generated and cyclic includes finite cyclic). But if $G$ is torsion-free then it has geometric dimension 2 so all finite index subgroups have deficiency 1 .

If we have a yes answer to this question then by [36] $H$, and so $G$, is coherent, which is a special case of the old question of whether all 1-relator groups are coherent.

Update. No progress in the 2-generator 1-relator case, but [5] shows that cyclically pinched 1-relator groups are often virtually free-by-cyclic (though the free subgroup will be infinitely generated for more than 2 generators).

21 (Borisov and Sapir [16]). Is the property of being residually finite generic amongst 2-generator 1-relator presentations?

Notes. It is shown in [31] Theorem 6.1 that a generic presentation is not an ascending HNN extension of a finitely generated free group, but experimentally $94 \%$ of presentations give groups of the form $F_{n} \rtimes \mathbb{Z}$. There is an algorithm, due to Moldavanskii [56] in the case $\beta_{1}(G)=1$ and Brown [20] for $\beta_{1}(G)=2$, as to whether a 2-generator 1-relator group is of this form. One could try looking for $H \leq_{f} G$ with $H$ an ascending HNN extension of a finitely generated free group (which is shown to be residually finite in [16]), but the problem is of course that although $H$ will have a deficiency 1 presentation, it will not in general be 2-generated (indeed as soon as $d\left(H / H^{\prime}\right) \geq 3$ it cannot be).

We can also ask about genericity for other properties: being linear, being coherent (as evidence for the general conjecture) and being large. It is known that a generic presentation gives rise to a group that is one ended, torsion-free and word hyperbolic, so if $G=F_{n} \rtimes \mathbb{Z}$ is large for word hyperbolic $G$ with $n \geq 2$ as in Question 18 then we would expect at least $94 \%$ of random presentations to give large groups. 
Update. Again no progress in this case, but [65] shows that residual finiteness is generic amongst $n$-generator 1-relator presentations for $n>2$ (indeed this is established by embedding the group into a 2-generator 1-relator group which is an ascending $\mathrm{HNN}$ extension of a finitely generated free group). As for these properties in the 2-generator 1-relator case, we suggest the following order for the likelihood of their genericity: first coherence as it could be that 2-generator 1-relator groups are coherent without exception (indeed as any finitely generated 1-relator group can be embedded in a 2-generator 1-relator group, this would establish coherence of all finitely generated 1-relator groups). Next largeness as 1-relator groups with 3 or more generators are large without exception, then residual finiteness as having 3 or more generators implies residual finiteness is a generic (but not universal) property. (Also we know of plenty of 2-generator 1-relator groups which are large but not residually finite, but only those in Question 18 for the other way round.) Finally linearity which implies residual finiteness.

22. Given reduced words $u, v \in F_{2}=\langle x, y\rangle$ which are not a generating pair, does there exist a homomorphism $\rho$ from $F_{2}$ into the symmetric group $\Sigma_{n}$ for some $n$ such that $\rho(u)$ and $\rho(v)$ commute, but $\rho(x)$ and $\rho(y)$ do not?

Notes. This is equivalent to asking: if the group $G=\langle x, y \mid[u, v]\rangle$ is proabelian then is it abelian (and thus equal to $\mathbb{Z} \times \mathbb{Z}$ )? Again there are interesting consequences because if so then $G$ is either large or $\mathbb{Z} \times \mathbb{Z}$ by [27] Theorem 3.6, but if not then [59], Question (OR8), is answered, which asks if $G$ is always residually finite.

23 (Müller and Schlage-Puchta [58], Problem 5). Is there an algorithm to detect whether a 2-generator 1-relator presentation is large?

This special case of Question 12 might be more likely to yield the answer yes. However even if there is a straightforward criterion for largeness of such presentations, it might not convert into an algorithm: for instance if Question 19 is true then it will detect largeness (which we can do anyway for an arbitrary finitely presented group) but would fail to prove that a given presentation is not large. We believe that Section 4 provides evidence that there is not an obvious algorithm.

\section{References}

[1] L.Bartholdi, On amenability of group algebras, II: graded algebras. Preprint 2006. arXiv:math/0611709v4 [math.GR]

[2] G. Baumslag, A non-cyclic one-relator group all of whose finite quotients are cyclic. $J$. Austral. Math. Soc. 10 (1969), 497-498. Zbl 0214.27402 MR 0254127

[3] G. Baumslag, Topics in combinatorial group theory. Lectures Math. ETH Zürich, Birkhäuser Verlag, Basel 1993. Zbl 0797.20001 MR 1243634 
[4] G. Baumslag, Some open problems. In Summer school in group theory in Banff, 1996, CRM Proc. Lecture Notes 17, Amer. Math. Soc., Providence, RI, 1999, 1-9. Zbl 1113.20305 MR 1653682

[5] G. Baumslag, B. Fine, C. F. Miller, III, and D. Troeger, Virtual properties of cyclically pinched one-relator groups. Internat. J. Algebra Comput. 19 (2009), 213-227. Zbl 05572763 MR 2512551

[6] G. Baumslag, C. F. Miller, III, and D. Troeger, Reflections on the residual finiteness of one-relator groups. Groups Geom. Dyn. 1 (2007), 209-219.Zbl 1141.20024 MR 2314044

[7] B. Baumslag and S. J. Pride, Groups with two more generators than relators. J. London Math. Soc. (2) 17 (1978), 425-426. Zbl 0387.20030 MR 0491967

[8] M. Bestvina, Questions in geometric group theory. http://www.math.utah.edu/ bestvina

[9] M. Bestvina and M. Feighn, A combination theorem for negatively curved groups. $J$. Differential Geom. 35 (1992), 85-101. Zbl 0724.57029 MR 1152226

[10] M. Bestvina and M. Feighn, Addendum and correction to: "A combination theorem for negatively curved groups" [9]. J. Differential Geom. 43 (1996), 783-788. Zbl 0862.57027 MR 1412684

[11] M. Bestvina and M. Handel, Train tracks and automorphisms of free groups. Ann. of Math. (2) 135 (1992), 1-51. Zbl 0757.57004 MR 1147956

[12] R. Bieri, Homological dimension of discrete groups. Queen Mary College Mathematical Notes, Queen Mary College Department of Pure Mathematics, London 1976. Zbl 0357.20027 MR 0466344

[13] R. Bieri, Deficiency and the geometric invariants of a group. J. Pure Appl. Algebra 208 (2007), 951-959. Zbl 1175.20044 MR 2283437

[14] R. Bieri, W. D. Neumann, and R. Strebel, A geometric invariant of discrete groups. Invent. Math. 90 (1987), 451-477. Zbl 0642.57002 MR 914846

[15] R. Bieri and R. Strebel, Almost finitely presented soluble groups. Comment. Math. Helv. 53 (1978), 258-278. Zbl 0373.20035 MR 0498863

[16] A. Borisov and M. Sapir, Polynomial maps over finite fields and residual finiteness of mapping tori of group endomorphisms. Invent. Math. 160 (2005), 341-356. Zbl 1083.14023 MR 2138070

[17] A. V. Borshchev and D. I. Moldavanskiĭ, On the isomorphism of some groups with one defining relation. Mat. Zametki 79 (2006), 34-44; English transl. Math. Notes 79 (2006), 31-40. Zbl 1137.20023 MR 2252133

[18] N. Brady and J. Crisp, CAT(0) and CAT(-1) dimensions of torsion free hyperbolic groups. Comment. Math. Helv. 82 (2007), 61-85. Zbl 1145.20023 MR 2296058

[19] P. Brinkmann, Hyperbolic automorphisms of free groups. Geom. Funct. Anal. 10 (2000), 1071-1089. Zbl 0970.20018 MR 1800064

[20] K. S. Brown, Trees, valuations, and the Bieri-Neumann-Strebel invariant. Invent. Math. 90 (1987), 479-504. Zbl 0663.20033 MR 914847

[21] K. S. Brown and R. Geoghegan, An infinite-dimensional torsion-free $\mathrm{FP}_{\infty}$ group. Invent. Math. 77 (1984), 367-381. Zbl 0557.55009 MR 752825 
[22] A. M. Brunner, A group with an infinite number of Nielsen inequivalent one-relator presentations. J. Algebra 42 (1976), 81-84. Zbl 0342.20014 MR 0419617

[23] A. M. Brunner, On a class of one-relator groups. Canad. J. Math. 32 (1980), 414-420. Zbl 0405.20033 MR 571934

[24] R. G. Burns, A. Karrass, and D. Solitar, A note on groups with separable finitely generated subgroups. Bull. Austral. Math. Soc. 36 (1987), 153-160. Zbl 0613.20018 MR 897431

[25] J. O. Button, Mapping tori with first Betti number at least two. J. Math. Soc. Japan 59 (2007), 351-370. Zbl 1124.57001 MR 2325689

[26] J. O. Button, A formula for the normal subgroup growth of Baumslag-Solitar groups. J. Group Theory 11 (2008), 879-884. Zbl 1153.20024 MR 2466914

[27] J. O. Button, Large groups of deficiency 1. Israel J. Math. 167 (2008), 111-140. Zbl 05508703 MR 2448020

[28] J. O. Button, Finite covers of the infinite cyclic cover of a knot. J. Knot Theory Ramifications 18 (2009), 75-85. Zbl 1165.57002 MR 2490018

[29] J. O. Button, Proving finitely presented groups are large by computer. Experiment. Math., To appear.

[30] N. M. Dunfield, Alexander and Thurston norms of fibered 3-manifolds. Pacific J. Math. 200 (2001), 43-58. Zbl 1049.57012 MR 1863406

[31] N. M. Dunfield and D. P. Thurston, A random tunnel number one 3-manifold does not fiber over the circle. Geom. Topol. 10 (2006), 2431-2499. Zbl 1139.57018 MR 2284062

[32] M. Edjvet, The concept of "largeness" in group theory. Ph.D. thesis, University of Glasgow, Glasgow 1984.

[33] M. Edjvet and S. J. Pride, The concept of "largeness" in group theory II. In GroupsKorea 1983 (Kyoungju, 1983), Lecture Notes in Math. 1098, Springer-Verlag, Berlin 1984, 29-54. Zbl 0566.20014 MR 0781355

[34] M. Ershov, Golod-Shafarevich groups with property $(T)$ and Kac-Moody groups. Duke Math. J. 145 (2008), 309-339. Zbl 1162.20018 MR 2449949

[35] M. Ershov and A. Jaikin-Zapirain, Kazhdan quotients of Golod-Shafarevich groups. Proc. London Math. Soc., to appear. arXiv:0908.3734v1[math.GR]

[36] M. Feighn and M. Handel, Mapping tori of free group automorphisms are coherent. Ann. of Math. (2) 149 (1999), 1061-1077. Zbl 0938.20022 MR 1709311

[37] E. Gelman, Subgroup growth of Baumslag-Solitar groups. J. Group Theory 8 (2005), 801-806. Zbl 1105.20018 MR 2179671

[38] S. M. Gersten, Isoperimetric and isodiametric functions of finite presentations. In Geometric group theory, (Sussex, 1991), Vol. 1, London Math. Soc. Lecture Note Ser. 181, Cambridge University Press, Cambridge 1993, 79-96. Zbl 0829.20054 MR 1238517

[39] S. M. Gersten and J. R. Stallings, Irreducible outer automorphisms of a free group. Proc. Amer. Math. Soc. 111 (1991), 309-314. Zbl 0717.20026 MR 1052571

[40] G. Higman, A finitely generated infinite simple group. J. London Math. Soc. 26 (1951), 61-64. Zbl 0042.02201 MR 0038348

[41] J. A. Hillman, Elementary amenable groups and 4-manifolds with Euler characteristic 0. J. Austral. Math. Soc. Ser. A 50 (1991), 160-170. Zbl 0733.57009 MR 1094067 
[42] J. A. Hillman, Tits alternatives and low dimensional topology. J. Math. Soc. Japan 55 (2003), 365-383. Zbl 1056.57002 MR 1961291

[43] C. H. Houghton, The first cohomology of a group with permutation module coefficients. Arch. Math. (Basel) 31 (1978), 254-258. Zbl 0377.20044 MR 521478

[44] J. Howie, Free subgroups in groups of small deficiency. J. Group Theory 1 (1998), 95-112. Zbl 0888.20018 MR 1490160

[45] S. C. Jeanes and J. S. Wilson, On finitely generated groups with many profinite-closed subgroups. Arch. Math. (Basel) 31 (1978), 120-122. Zbl 0377.20029 MR 512725

[46] E. I. Khukhro, A. A. Klyachko, N. Y. Makarenko, and Y. B. Melnikova, Automorphism invariance and identities. Bull. Lond. Math. Soc. 41 (2009), 804-816. Zbl 05636218 MR 2557461

[47] D. H. Kochloukova, Some Novikov rings that are von Neumann finite and knot-like groups. Comment. Math. Helv. 81 (2006), 931-943. Zbl 1166.20042 MR 2271229

[48] V. D. Mazurov and E. I. Khukhro (eds.), The Kourovka notebook. Unsolved problems in group theory. 16th ed., Russian Academy of Sciences, Siberian Division, Institute of Mathematics, Novosibirsk 2006. Zbl 1084.20001 MR 2263886

[49] D. D. Long and A. W. Reid, Subgroup separability and virtual retractions of groups. Topology 47 (2008), 137-159. Zbl 1169.57003 MR 2414358

[50] A. Lubotzky, Discrete groups, expanding graphs and invariant measures. Progr. Math. 125, Birkhäuser Verlag, Basel 1994. Zbl 0826.22012 MR 1308046

[51] A. Lubotzky, Free quotients and the first Betti number of some hyperbolic manifolds. Transform. Groups 1 (1996), 71-82. Zbl 0876.22015 MR 1390750

[52] A. Lubotzky and A. Mann, Residually finite groups of finite rank. Math. Proc. Cambridge Philos. Soc. 106 (1989), 385-388. Zbl 0696.20031 MR 1010362

[53] R. C. Lyndon and P. E. Schupp, Combinatorial group theory. Ergeb. Math. Grenzgeb. 89, Springer-Verlag, Berlin 1977. Zbl 0368.20023 MR 0577064

[54] W. Magnus, A. Karrass, and D. Solitar, Combinatorial group theory. 2nd ed., Dover Publications Inc., Mineola, NY, 2004. Zbl 1130.20307 MR 2109550

[55] V. Metaftsis and E. Raptis, Subgroup separability of HNN-extensions with abelian base group. J. Algebra 245 (2001), 42-49. Zbl 0999.20018 MR 1868182

[56] D. I. Moldavanskii, Certain subgroups of groups with a single defining relation. Sibirsk. Mat. Zh. 8 (1967), 1370-1384; English transl. Siberian Math. J. 8 (1967), 1039-1048. Zbl 0169.33602 MR 0220810

[57] D. Moldavanski and N. Sibyakova, On the finite images of some one-relator groups. Proc. Amer. Math. Soc. 123 (1995), 2017-2020. Zbl 0834.20029 MR 1273511

[58] T. W. Müller and J.-C. Schlage-Puchta, Some examples in the theory of subgroup growth. Monatsh. Math. 146 (2005), 49-76. Zbl 1127.20022 MR 2167869

[59] New York Group Theory Cooperative, Open problems in combinatorial and geometric group theory. Available at http://www.sci.ccny.cuny.edu/ shpil/gworld/problems/oproblems.html

[60] Y. Ollivier and D. T. Wise, Kazhdan groups with infinite outer automorphism group. Trans. Amer. Math. Soc. 359 (2007), 1959-1976. Zbl 1176.20031 MR 2276608 
[61] A. Yu. Ol'shanskii, Geometry of defining relations in groups. Math Appl. (Soviet Series) 70, Kluwer Academic Publishers, Dordrecht 1991. Zbl 0732.20019 MR 1191619

[62] A. Yu. Ol'shanskiŭ, SQ-universality of hyperbolic groups. Mat. Sb. 186 (1995), 119-132; English transl. Sb. Math. 186 (1995), no. 8, 1199-1211. Zbl 0864.20023 MR 1357360

[63] A. Yu. Olshanskii and M. V. Sapir, Non-amenable finitely presented torsion-by-cyclic groups. Publ. Math. Inst. Hautes Études Sci. 96 (2002), 43-169. Zbl 1050.20019 MR 1985031

[64] D. J. S. Robinson, A course in the theory of groups. 2nd ed., Graduate Texts in Math. 80, Springer-Verlag, New York 1996. Zbl 0836.20001 MR 1357169

[65] M. Sapir and I. Špakulová, Almost all one-relator groups with at least three generators are residually finite. J. Eur. Math. Soc., to appear. arXiv:0809.4693v2 [math.GR]

[66] J. R. Stallings, Topologically unrealizable automorphisms of free groups. Proc. Amer. Math. Soc. 84 (1982), 21-24. Zbl 0477.20012 MR 633269

[67] J. S. Wilson, Finitely presented soluble groups. In Geometry and cohomology in group theory (Durham, 1994), London Math. Soc. Lecture Note Ser. 252, Cambridge Univ. Press, Cambridge 1998, 296-316. Zbl 0908.20025 MR 1709964

[68] E. Zelmanov, On groups satisfying the Golod-Shafarevich condition. In New horizons in pro-p groups, Progr. Math. 184, Birkhäuser, Boston 2000, 223-232. Zbl 0974.20022 MR 1765122

Received March 24, 2008; revised January 20, 2010

J. O. Button, Selwyn College, University of Cambridge, Cambridge CB3 9DQ, UK

E-mail: jb128@dpmms.cam.ac.uk 